SGLT2 inhibitors for the prevention of kidney failure in patients with type 2 diabetes: a systematic review and meta-analysis

Brendon L. Neuen MBBS(Hons) ${ }^{1}$, Tamara Young MBBS(Hons) ${ }^{1}$, Prof Hiddo J.L. Heerspink $\mathrm{PhD}^{1,2}$, Prof Bruce Neal $\mathrm{PhD}^{1,3,4}$, Prof Vlado Perkovic PhD ${ }^{1}$, Laurent Billot MSc ${ }^{1}$, Prof Kenneth W. Mahaffey MD ${ }^{5}$, David M. Charytan $\mathrm{MD}^{6}$, Prof David C. Wheeler MD, ${ }^{7}$ Clare Arnott $\mathrm{PhD}^{1,3,8}$, Severine Bompoint $\mathrm{BSc}^{1}$, Prof Adeera Levin $\mathrm{MD}^{9}$, Meg J. Jardine $\mathrm{PhD}^{1,10}$

${ }^{1}$ The George Institute for Global Health, UNSW Sydney, Australia; ${ }^{2}$ University of Groningen, University Medical Center Groningen, Groningen, The Netherlands; ${ }^{3}$ The Charles Perkins Centre, University of Sydney, Sydney, Australia; ${ }^{4}$ Epidemiology and Biostatistics, Imperial College London, London, UK; ${ }^{5}$ Stanford Center for Clinical Research, Department of Medicine, ${ }^{5}$ Stanford University School of Medicine, Stanford University, Stanford, USA; ${ }^{6}$ Nephrology Division, Department of Medicine, NYU Langone Medical Center, New York, USA; ${ }^{7}$ Department of Renal Medicine, University College London, London, United Kingdom, ${ }^{8}$ Department of Cardiology, Royal Prince Alfred Hospital, Sydney, Australia; ${ }^{9}$ Division of Nephrology, University of British Columbia, Vancouver, Canada; ${ }^{10}$ Concord Repatriation and General Hospital, Sydney, Australia

Manuscript type: Systematic review and meta-analysis

Word count: 4154

Abstract word count: 361

Running title: SGLT2 inhibitors and kidney failure

Keywords: SGLT2 inhibitors, type 2 diabetes, renal outcomes, systematic review, meta-analysis

Corresponding author: Associate Professor Meg Jardine

Address: Level 5, 1 King Street Newtown NSW 2042 Australia

Phone: +61 280524300 Fax: +6128052 4301

Email: mjardine@georgeinstitute.org.au 


\section{RESEARCH IN CONEXT}

\section{Evidence before this study}

Large-scale randomized cardiovascular outcome trials of sodium glucose cotransporter-2 (SGLT2) inhibitors have suggested promising effects on albuminuria and creatinine based kidney outcomes. However, these trials included few participants at high risk of clinically important kidney outcomes, and as a result, the effect of SGLT2 inhibitors on kidney outcomes of greatest concern to patients namely, the need for long-term dialysis, transplantation or death due to kidney disease - has been uncertain. SGLT2 inhibitors are also not currently approved for use in patients with estimated glomerular filtration rate (eGFR) $<45$ or $60 \mathrm{~mL} / \mathrm{min} / 1 \cdot 73 \mathrm{~m}^{2}$ in most countries, primarily because their glucose lowering effect is substantially dependent on kidney function. A recent meta-analysis of these trials has reported that the renoprotective effect of SGLT2 attenuates with declining kidney function. However, less than a sixth of participants studied had a baseline eGFR less than 60 $\mathrm{mL} / \mathrm{min} / 1.73 \mathrm{~m}^{2}$, and even fewer had baseline eGFR $<45 \mathrm{~mL} / \mathrm{min} / 1.73 \mathrm{~m}^{2}$. The ability to robustly assess effects in people with reduced kidney function was therefore limited, especially since few patient-level kidney outcomes occurred. While collectively these trials have suggested that SGLT2 inhibitors might protect against acute kidney injury, the safety of these agents in patients at high risk of adverse kidney outcomes has remained a concern. More recently the CREDENCE trial (Canagliflozin and Renal Endpoints in Diabetes with Established Nephropathy Clinical Evaluation) has been published, which was designed specifically to examine the impact of SGLT2 inhibition in people at high risk of kidney disease progression. We therefore conducted a systematic review and meta-analysis of randomized, controlled, event-driven, cardiovascular and kidney outcome trials reporting effects of major kidney outcomes in patients with type 2 diabetes. MEDLINE and EMBASE were searched from inception until June 142019 to identify potentially relevant studies. The protocol for this systematic review and meta-analysis was registered in the International Prospective Register of Systematic Reviews before the analyses were completed (PROSPERO registration number CRD42019131774). 


\section{Added value of this study}

This review summarises data from four studies including 38,723 participants across six continents. There was clear evidence that SGLT2 inhibitors reduce the risk dialysis, transplantation or death due to kidney disease, as well as a range of other major kidney outcomes, and that these agents also provide protection against acute kidney injury. Additionally, there were definitive, separate benefits at all levels of kidney function, including an approximate $30 \%$ proportional risk reduction in the composite kidney outcome in participants with baseline eGFR less than $45 \mathrm{~mL} / \mathrm{min} / 1 \cdot 73 \mathrm{~m}^{2}$ in whom these drugs are mostly not permitted for use.

\section{Implications of all the available evidence}

These results provide the strongest evidence yet that SGLT2 inhibitors should be routinely offered to individuals with type 2 diabetes at risk of progressive kidney disease. The clear evidence of renoprotection across the spectrum of kidney function studied to date call into question current restrictions on the use of SGLT2 inhibitors in people with reduced kidney function, and suggest that many more individuals with type 2 diabetes at high risk of kidney failure are likely to benefit from treatment. 


\section{SUMMARY}

\section{Background}

The effect of sodium-glucose cotransporter 2 (SGLT2) inhibitors on kidney failure, particularly the need for dialysis and transplantation, or death due to kidney disease, has been uncertain. Previous studies have also been underpowered to robustly assess heterogeneity of effects on kidney outcomes by different levels of estimated glomerular filtration rate (eGFR) and albuminuria.

\section{Methods}

We conducted a systematic review and meta-analysis of randomized, controlled, cardiovascular or kidney outcome trials of SGLT2 inhibitors reporting effects on major kidney outcomes in people with type 2 diabetes (PROSPERO registration number CRD42019131774). MEDLINE and EMBASE were searched from inception to 14 June 2019 to identify eligible trials. The primary outcome was dialysis, transplantation, or death due to kidney disease. We used random effects models to obtain summary relative risks (RR) with $95 \%$ confidence intervals $(\mathrm{CI})$ and random effects meta-regression to explore effect modification by subgroups of baseline eGFR, albuminuria, and use of renin-angiotensin system (RAS) blockade.

\section{Findings}

Data were obtained from four studies including 38,723 participants of whom 252 required dialysis or transplantation or died due to kidney disease, 335 developed end-stage kidney disease (ESKD), and 943 experienced acute kidney injury (AKI). SGLT2 inhibitors reduced the risk of dialysis, transplantation or death due to kidney disease (RR 0.67, 95\% CI 0.52-0.86, $p=0 \cdot 0019), \mathrm{ESKD}(\mathrm{RR}$ $0 \cdot 65,95 \%$ CI $0 \cdot 53-0 \cdot 81, p<0 \cdot 0001)$, and AKI (RR 0.75, 95\% CI 0.66-0.85, $p<0 \cdot 0001)$, with consistent benefits across studies. While there was some evidence that the proportional effect of SGLT2 inhibitors might attenuate with declining kidney function $(P$-trend $=0 \cdot 073)$, there was clear, separate evidence of benefit for all eGFR subgroups, including for participants with starting eGFR $30-45 \mathrm{~mL} / \mathrm{min} / 1 \cdot 73 \mathrm{~m}^{2}(\mathrm{RR} 0 \cdot 70,95 \%$ CI $0 \cdot 54-0 \cdot 91, p=0 \cdot 0080)$. Renoprotection was also 
consistent irrespective of baseline albuminuria and use of RAS blockade $(P$-trend $=0 \cdot 66$ and $P$ heterogeneity $=0 \cdot 31$, respectively).

\section{Interpretation}

SGLT2 inhibitors reduce the risk of dialysis, transplant or death due to kidney disease and provide protection against acute kidney injury. These data provide substantive evidence supporting the use of SGLT2 inhibitors to prevent major kidney outcomes in people with type 2 diabetes.

\section{Funding}

None. 


\section{INTRODUCTION}

It is estimated that approximately 2.6 million people received dialysis or underwent kidney transplantation for kidney failure in 2010, and this number is projected to more than double by 2030. ${ }^{1}$ In many countries, more than half of all patients entering dialysis programmes have type 2 diabetes mellitus (T2DM), making it a leading cause of kidney failure worldwide. ${ }^{2}$ Kidney failure due to T2DM is a large and growing challenge, not only for patients and their families and caregivers, but also for health systems and governments. ${ }^{3}$

Treatment with angiotensin converting enzyme (ACE) inhibitors or angiotensin-receptor blockers (ARBs) has been shown to prevent major kidney outcomes in people with diabetes, and these agents are currently recommended by clinical practice guidelines for the treatment of people with T2DM who have, or are at high risk of, kidney disease. ${ }^{4-8}$ However, the residual risk remains high with new treatments urgently needed to reduce the growing burden of kidney failure.

SGLT2 inhibitors are a newer class of glucose-lowering agent that also lower blood pressure, body weight and albuminuria, and may have direct haemodynamic effects on the kidney. ${ }^{9}$ Large scale randomized trials of SGLT2 inhibitors, which were originally designed to meet regulatory requirements and ensure cardiovascular safety, have demonstrated promising effects on a range of albuminuria and serum creatinine based kidney outcomes in patients with, or at high risk of, atherosclerotic cardiovascular disease. ${ }^{10-15}$ The majority of participants in these trials were at low risk of clinically important kidney outcomes, and as a result, event rates for kidney failure were low, with few participants requiring dialysis or kidney transplantation, or dying due to kidney disease, in each trial. As they were also not explicitly designed to provide definitive information on renoprotective effects, kidney endpoints were not always pre-specified, were not always adjudicated, and the distinction between acute and chronic reductions in estimated glomerular filtration rate (eGFR) was not possible in all studies. 
A recent meta-analysis of cardiovascular outcome trials suggested that the effect of SGLT2 inhibitors on kidney outcomes attenuates with declining eGFR. ${ }^{14}$ However, less than a sixth of participants in this analysis had baseline eGFR $<60 \mathrm{~mL} / \mathrm{min} / 1 \cdot 73 \mathrm{~m}^{2}$, and thus the ability to robustly assess effect modification by kidney function has been limited. Additionally, because very few participants with starting eGFR $<45 \mathrm{~mL} / \mathrm{min} / 1 \cdot 73 \mathrm{~m}^{2}$ have been studied, it has also been unclear whether these patients derive protection against kidney outcomes, since the glycaemic efficacy of these agents is substantially attenuated in this population. Similarly, few participants with higher levels of albuminuria have been studied, and therefore the consistency of treatment effect across different levels of albuminuria is unclear. While these trials have collectively suggested protection against acute kidney injury, ${ }^{16}$ the safety of these agents in patients at higher risk of adverse kidney outcomes has not been well established. Most recently, the Canagliflozin and Renal Endpoints in Diabetes with Established Nephropathy Clinical Evaluation (CREDENCE) trial has been reported, which is the first study designed to specifically evaluate the impact of an SGLT2 inhibitor on a primary kidney outcome in people with established diabetic kidney disease. ${ }^{17}$

We therefore undertook a systematic review and meta-analysis to determine the consistency of effect size across SGLT2 trials and different levels of kidney function and albuminuria, summarize results, and integrate available data on the effects of SGLT2 inhibition on the risk of clinically important kidney outcomes in people with T2DM.

\section{METHODS}

This systematic review and meta-analysis was conducted and reported using the Preferred Reporting Items for Systematic Reviews and Meta-Analysis (PRISMA) statement. The protocol for this review was submitted before the analyses were begun (10 April 2019) and was registered in the International Prospective Register of Systematic Reviews before the analyses were completed 
(PROSPERO registration number CRD42019131774) and can be accessed

at: http://www.crd.york.ac.uk/PROSPERO/display_record.php?ID=CRD42019131774.

\section{Search strategy and selection criteria}

We searched MEDLINE and EMBASE from inception to 14 June 2019 to identify potentially eligible studies. Details of the search strategy, including text words and medical subject headings are provided in Table S1. We included all randomized, controlled, event-driven, cardiovascular or kidney outcome trials of SGLT2 inhibitors versus active or placebo control, in order to capture those with meaningful numbers of clinical kidney outcomes. Trials with extension periods and those including participants with type 1 diabetes or individuals less than 18 years of age were excluded. Two authors (B.L.N and T.Y) independently screened the titles and abstracts of all identified articles and, when required, reviewed full-text manuscripts to identify potentially relevant studies. Any disagreements related to the identification or eligibility of studies was resolved through discussion with a third author (M.J.J). Study sponsor and investigators were contacted to obtain additional trial-level data and clarify outcome definitions when required.

\section{Data synthesis and analysis}

Two authors (B.L.N and T.Y) independently extracted all data using a standardized data form and assessed risk of bias at the study level using the Cochrane Risk of Bias Tool. ${ }^{18}$ We used image extraction software to extract data presented only in figures without corresponding numerical data (WebPlotDigitizer version 4·1, Ankit Rohatgi, Austin, TX, https://automeris.io/WebPlotDigitizer/). These data were summarized descriptively and not used for quantitative synthesis. Any discrepancies in data extraction or risk of bias assessment were resolved in consultation with a third author (M.J.J). Due to the small number of eligible trials, publication bias was not assessed. 
The primary outcome of interest was chronic dialysis, kidney transplantation, or death due to kidney disease. Other kidney outcomes included: (1) ESKD, defined as chronic dialysis, kidney transplantation, or sustained eGFR less than $15 \mathrm{~mL} / \mathrm{min} / 1 \cdot 73 \mathrm{~m}^{2}$, (2) substantial loss of kidney function, ESKD, or death due to kidney disease, (3) substantial loss of kidney function, ESKD, or death due to cardiovascular or kidney disease, (4) long-term eGFR slope, and (5) acute kidney injury (AKI). Substantial loss of kidney function was preferentially defined as a sustained doubling of serum creatinine (representing an approximate 57\% decline in kidney function). Where sustained doubling of serum creatinine was not reported, we included sustained $40 \%$ decline in eGFR or unsustained doubling of serum creatinine as defined by study authors. We preferentially used data on sustained kidney outcomes confirmed with repeat assessment where these were reported to exclude acute changes in kidney function and initiation of dialysis for AKI, but accepted unsustained outcomes where these were the only ones reported. This was the case for the EMPAREG OUTCOME trial, where sustained kidney outcome data were reported in a separate correspondence ${ }^{19}$ following the main trial publication. ${ }^{11}$ The definitions of long-term eGFR slope (annualized difference in eGFR between treatment and control) and AKI varied across studies and we used these outcomes as defined and reported in each study.

We prespecified that results for dichotomous outcomes were to be quantitatively synthesized by individual studies using a random effects model with inverse variance weighting to obtain summary effect estimates represented as relative risk (RR) with associated 95\% confidence intervals $(\mathrm{CI})$. We also decided a priori to pool, in order of preference, hazard ratios, incidence rate ratios (events/patient-years), and risk ratios (events/number of participants) to maximize the use of trial level data from included studies, particularly for canagliflozin, where the integrated analysis and reporting of two parallel companion trials with different randomization ratios and different followup durations precluded the use of risk ratios. ${ }^{20}$ When studies did not report the preferred outcome definition for substantial loss of kidney function, we performed sensitivity tests excluding those 
studies to assess the impact of endpoint definition on the results. We prospectively decided to summarize the effect of SGLT2 inhibitors on long-term eGFR slope descriptively because of variations in the definition of this outcome and because it measured the absolute rather than proportional effect of treatment. Any heterogeneity between studies for this outcome therefore could not be meaningfully assessed, as differences in absolute effect reflected differences in baseline kidney risk. For all other outcomes we assessed heterogeneity between studies using the $\mathrm{I}^{2}$ test and $P$-heterogeneity values obtained from a random effects model. $\mathrm{I}^{2}$ values of $<25 \%, 25-75 \%$, and $>75-100 \%$ were considered to reflect low, moderate, and high likelihood of differences between studies, respectively.

Because of the kidney-based mechanism of action and albuminuria lowering effect of SGLT2 inhibitors, we prospectively decided to conduct subgroup analyses for efficacy outcomes to assess for effect modification by three kidney-related subgroups. The effect of SGLT2 inhibitors on the composite kidney outcome of substantial loss of kidney function, ESKD or death due to kidney disease was assessed across different levels of baseline kidney function and urinary albumin excretion. Additionally, because ACE inhibitors and ARBs are recommended for the treatment of diabetic kidney disease, we also analysed whether the effects of SGLT2 inhibition differed by baseline use of renin-angiotensin system (RAS) blockade. Because the results come from relatively few individual studies, we performed multiple sensitivity analyses to assess the vulnerability of the eGFR subgroup analysis outcomes to definitional and methodological decisions. We assessed the impact of SGLT2 inhibitors by eGFR categories (eGFR $<45,45-<60,60-<90$ and $\geq 90$ $\mathrm{mL} / \mathrm{min} / 1 \cdot 73 \mathrm{~m}^{2}$ ) and levels of albuminuria (urinary albumin:creatinine ratio [UACR] $<30,30-300$, and $>300 \mathrm{mg} / \mathrm{g}$ ) as the main analysis. Where studies reported the eGFR $<60 \mathrm{~mL} / \mathrm{min} / 1 \cdot 73 \mathrm{~m}^{2}$ subgroup without more granular categories, as occurred in the DECLARE-TIMI 58 trial, we excluded these data from the main analysis. However we performed a sensitivity analysis in which the outcomes for the baseline eGFR $<60 \mathrm{~mL} / \mathrm{min} / 1 \cdot 73 \mathrm{~m}^{2}$ subgroup were included with the eGFR 45 - 
$<60 \mathrm{~mL} / \mathrm{min} / 1 \cdot 73 \mathrm{~m}^{2}$ category, on the assumption that a large majority of these participants were likely to have an eGFR $45-60 \mathrm{~mL} / \mathrm{min} / 1.73 \mathrm{~m}^{2}$ based on the trial exclusion criteria. We conducted an additional sensitivity analysis to assess the effects of treatment in participants with eGFR $<60$ and $\geq 60 \mathrm{~mL} / \mathrm{min} / 1 \cdot 73 \mathrm{~m}^{2}$. When required, effect estimates for subgroups within the same study (e.g. eGFR $30-<45$ and $45-<60 \mathrm{~mL} / \mathrm{min} / 1 \cdot 73 \mathrm{~m}^{2}$ ) were merged using a fixed effects model. For the eGFR slope outcome, data were stratified by kidney function (eGFR $<60$ and $\geq 60 \mathrm{~mL} / \mathrm{min} / 1 \cdot 73 \mathrm{~m}^{2}$ ) and albuminuria (UACR $<30,30-300$, and $>300 \mathrm{mg} / \mathrm{g}$ ) and summarized descriptively.

We decided a priori to use random effects meta-regression to assess trend in treatment effects across eGFR and albuminuria subgroups as the primary analysis. In sensitivity analyses we repeated the meta-regression analyses treating subgroups as categories without assumptions of linearity. A $P$ trend and $P$-heterogeneity of $<0 \cdot 10$ were considered to reflect a high likelihood of differences beyond chance.

All analyses were performed using Stata 15·1 (StataCorp 2017. Stata Statistical Software: Release 15. College Station, TX; StataCorp LLC).

\section{Role of the funding source}

This study was not specifically funded. All authors had full access to the data and agreed on the final decision to submit for publication.

\section{RESULTS}

We identified four separate studies comprising five individual trials after applying the search strategy and study selection criteria (Table S1 and Figure S1). Data from a number of secondary analyses of these studies were used in this meta-analysis. ${ }^{11-13,15,19,21-24}$ One secondary analysis of the DECLARE-TIMI 58 trial which was published after the systematic literature search was identified 
by hand searching. ${ }^{15}$ The CANVAS Program comprised two companion trials (CANVAS and CANVAS-R) that were conducted in parallel and analysed and reported as a single program. ${ }^{25}$ All studies compared an SGLT2 inhibitor with matching placebo. Three studies were designed as cardiovascular outcome trials testing the impact of empagliflozin, canagliflozin, and dapagliflozin on a primary composite cardiovascular outcome of nonfatal myocardial infarction, nonfatal stroke, or cardiovascular death, with a range of pre-specified exploratory and post-hoc kidney outcomes also reported. ${ }^{25-27}$ One study was an event-driven kidney outcome trial for canagliflozin with a primary composite outcome of sustained doubling of serum creatinine, ESKD, or death due to cardiovascular or kidney disease. ${ }^{17}$ The risk of bias was low for all indicators; all participants and investigators were blinded to treatment allocation with complete reporting of outcomes (Figure S2).

In total, this meta-analysis included data on 38,723 randomised participants from six continents. The mean age across the studies ranged from 61.3 to 63.9 years, while $35.0 \%$ of participants overall were female (Table 1). The proportion of participants with eGFR $<60 \mathrm{~mL} / \mathrm{min} / 1 \cdot 73 \mathrm{~m}^{2}$ ranged from $7.4 \%$ in DECLARE-TIMI 58 to $58.9 \%$ in CREDENCE. The majority of participants in the three cardiovascular outcome trials had a UACR $<30 \mathrm{mg} / \mathrm{g}$ at baseline (range $59.4 \%$ to 69.1\%), while UACR $>300 \mathrm{mg} / \mathrm{g}$ was an entry criterion for the CREDENCE trial (Table 1). An eGFR of $\geq 30 \mathrm{~mL} / \mathrm{min} / 1 \cdot 73 \mathrm{~m}^{2}$ was an inclusion criterion in all studies with the exception of the DECLARE-TIMI 58 trial where Cockroft Gault creatinine clearance of $\geq 60 \mathrm{~mL} / \mathrm{min}$ was required. Treatment with RAS blockade was required for entry by the CREDENCE trial only. Accordingly, virtually all (99.9\%) CREDENCE participants were treated with ACE inhibitors or ARBs at baseline compared with approximately $80 \%$ of participants in the other trials (Table 1).

Overall, there were 252 participants who required dialysis or transplantation or died due to kidney disease. There were 335 ESKD events; 967 incidences of substantial loss of kidney function, ESKD or death due to kidney disease; 2,323 cases of substantial loss of kidney function, ESKD, or death 
due to cardiovascular or kidney disease; and 943 episodes of AKI. Pre-specification of outcomes, requirements for changes in kidney function to be confirmed on repeated measurement, and adjudication procedures differed across the studies (Table S3). Kidney endpoints were also defined and reported variably across the studies (Table S4-S5).

SGLT2 inhibitors reduced the risk of dialysis, transplantation or death due to kidney disease by $33 \%($ RR $0 \cdot 67,95 \%$ CI $0 \cdot 52-0 \cdot 86, p=0 \cdot 0019)$. The effect of SGLT2 inhibitors on this outcome was consistent across studies $\left(\mathrm{I}^{2}=0 \%, P\right.$-heterogeneity $=0 \cdot 53$; Figure 1$)$.

SGLT2 inhibitors reduced the risk of ESKD by $35 \%$ (RR 0.65, 95\% CI 0.53-0.81, $p<0 \cdot 0001$ ), with no differences in treatment effect across studies $\left(\mathrm{I}^{2}=0 \cdot 0 \%, P\right.$-heterogeneity $\left.=0 \cdot 41\right)$. SGLT2 inhibition also reduced the risk of substantial loss of kidney function, ESKD, or death due to kidney disease by $42 \%(0 \cdot 58,95 \%$ CI $0 \cdot 51-0 \cdot 66, \mathrm{p}<0 \cdot 0001$; Figure 2$)$ with no evidence of differences between studies $\left(\mathrm{I}^{2}=0 \%, P\right.$-heterogeneity=0 49). The results from sensitivity testing which excluded studies that did not report our preferred event definition of substantial loss of kidney function were essentially unchanged (Table S6). The overall effect of SGLT2 inhibitors on substantial loss of kidney function, ESKD, death due to cardiovascular or kidney disease (RR 0·71, 95\% CI 0.0.63$0 \cdot 82, p<0 \cdot 0001$; Figure 2) varied across studies, primarily due to the EMPA-REG OUTCOME trial $\left(\mathrm{I}^{2}=60 \cdot 3 \%, P\right.$-heterogeneity $\left.=0 \cdot 056\right)$.

SGLT2 inhibitors also lowered the risk of AKI by $25 \%$ (RR $0 \cdot 75,95 \%$ CI $0 \cdot 66-0 \cdot 85, p<0 \cdot 0001$; Figure 4$)$, with no evidence of differences between studies $\left(\mathrm{I}^{2}=0 \%, P\right.$-heterogeneity $\left.=0 \cdot 68\right)$. AKI events, both serious and non-serious, were reported variably across individual trials and were not adjudicated (Table S5). Overall effects of SGLT2 inhibition on major kidney outcomes are summarised in Figure 5. 
The effect on the outcome of substantial loss of kidney function, ESKD or death due to kidney disease was reported according to eGFR and UACR subgroups, and according to baseline use of RAS blockade in all four studies. There was some evidence that the magnitude of relative benefit might be attenuated across progressively lower eGFR subgroups $(P$-trend=0・073; Figure 3$)$.

However, there was still clear, separately significant evidence of benefit for all eGFR subgroups, including for participants with a baseline eGFR $<45 \mathrm{~mL} / \mathrm{min} / 1 \cdot 73 \mathrm{~m}^{2}$, where a $30 \%$ relative risk reduction was observed $(\operatorname{RR} 0 \cdot 70,95 \% \mathrm{CI} 0 \cdot 54-0 \cdot 91, p=0 \cdot 0080)$. There were also clear and consistent benefits in participants with eGFR above and below $60 \mathrm{~mL} / \mathrm{min} / 1 \cdot 73 \mathrm{~m}^{2}(P$ heterogeneity=0 33; Figure S2). Results for tests of heterogeneity altered slightly in the different sensitivity analyses (Table S7) but the evidence of clear separate benefit for all eGFR subgroups remained constant. There was no evidence of differences in treatment effect for the composite outcome across UACR subgroups $(P$-trend=0·66; Figure 3$)$. The effect of SGLT2 inhibitors was also consistent irrespective of the use of renin-angiotensin system blockade at baseline $(P$ heterogeneity=0·31; Figure 3).

The absolute effect of SGLT2 inhibitors on annualised long-term eGFR slope is summarised in Table S8. The DECLARE-TIMI 58 trial reported mean eGFR over time as a prespecified outcome instead of annualised eGFR slope. The rate of eGFR decline in placebo treated participants varied between trials from $-0.85 \mathrm{~mL} / \mathrm{min} / 1.73 \mathrm{~m}^{2} /$ year in the CANVAS Program to -4.59

$\mathrm{mL} / \mathrm{min} / 1.73 \mathrm{~m}^{2} /$ year in the CREDENCE trial. As a result, annual placebo-subtracted differences in eGFR also differed, with the greatest absolute benefit in terms of eGFR decline observed in CREDENCE trial $\left(2 \cdot 74 \mathrm{~mL} / \mathrm{min} / 1 \cdot 73 \mathrm{~m}^{2} /\right.$ year, $95 \%$ CI $\left.2 \cdot 37-3 \cdot 11, p<0 \cdot 0001\right)$.

\section{DISCUSSION}

The development of kidney failure is among the most important consequences of diabetic kidney disease, and is of great concern to patients. The evidence from completed trials summarised in this 
review clearly shows that SGLT2 inhibitors definitively reduce the risk of dialysis, transplantation or death due to kidney disease, with compelling evidence of benefits on a broad range of other clinically important kidney outcomes. Importantly, renoprotection is achieved across all levels of kidney function down to eGFR $30 \mathrm{~mL} / \mathrm{min} / 1 \cdot 73 \mathrm{~m}^{2}$, with clear benefits even for the subgroup with baseline eGFR between 30 to $45 \mathrm{~mL} / \mathrm{min} / 1 \cdot 73 \mathrm{~m}^{2}$ in whom these drugs are currently not approved for use in most countries. The clear protective effect against AKI allays early concerns about the risk of adverse effects consequent upon the haemodynamic mechanism of action of this class of agent. Furthermore, the inclusion of CREDENCE, a trial that mandated the use of RAS blockade, shows that the benefits of SGLT2 inhibitors are cumulative with those of RAS blockade. These results provide the strongest evidence yet that SGLT2 inhibition should be routinely offered to individuals with T2DM at risk of progressive kidney disease.

The glycaemic efficacy of SGLT2 inhibitors is directly proportional to glomerular filtration rate, 9,28 but whether the renoprotective effects are modified by declining kidney function has been less clear. A recent meta-analysis of the cardiovascular outcome trials suggested that the renoprotective effect of SGLT2 inhibitors attenuates with declining kidney function. ${ }^{14}$ However too few participants with baseline eGFR $<60 \mathrm{~mL} / \mathrm{min} / 1.73 \mathrm{~m}^{2}$ were studied to adequately assess trend by eGFR. Additionally, whether participants with starting eGFR $<45 \mathrm{~mL} / \mathrm{min} / 1.73 \mathrm{~m}^{2}$ derive benefits with respect to kidney outcomes, despite substantially attenuated glycaemic efficacy, has also been unclear. The accumulated trial evidence, including the CREDENCE trial in which approximately $60 \%$ of participants had a baseline eGFR $<60 \mathrm{~mL} / \mathrm{min} / 1 \cdot 73 \mathrm{~m}^{2}$, has made it possible to robustly explore possible modifying effects. While our findings raise the possibility that the magnitude of relative (but not absolute) benefit might attenuate somewhat across progressively lower eGFR subgroups, these results clearly demonstrate that renoprotection is achieved across the entire spectrum of eGFR levels studied to date, down to an eGFR of $30 \mathrm{~mL} / \mathrm{min} / 1 \cdot 73 \mathrm{~m}^{2}$. SGLT2 inhibitors are currently not indicated in people with eGFR $<45 \mathrm{ml} / \mathrm{min} / 1 \cdot 73 \mathrm{~m}^{2}$ in most countries, 
largely due to limited glycaemic efficacy. ${ }^{29}$ As these individuals are at much greater risk of kidney failure, the absolute benefits of SGLT2 inhibition are likely to be at least as large as for people with higher eGFR. ${ }^{30}$ With evidence of renoprotection now available from the cumulated trials, these restrictions are called into question, suggesting that many more individuals at high risk of major kidney outcomes are likely to benefit from treatment, and that trials in people with even more advanced kidney disease are warranted. The absence of effect modification by baseline albuminuria contrasts with the findings from trials of RAS blockade. ${ }^{31-34}$ These data suggest that mechanisms other than those associated with albuminuria reduction might also be important. Furthermore, renoprotection with SGLT2 inhibitors appears consistent irrespective of baseline use of RAS blockade. Taken together, these findings suggest that SGLT2 inhibition should provide benefit for a broader patient population.

A plausible mechanistic explanation for the renoprotective effect of SGLT2 inhibitors is correction of aberrant glomerular haemodynamics induced by hyperglycaemia, which drive progressive nephron loss. ${ }^{35,36}$ Blocking sodium re-uptake in the proximal tubule has been suggested to restore delivery of sodium to the macula densa, leading to afferent arteriolar constriction and a reduction in intraglomerular pressure. ${ }^{37}$ This haemodynamic effect results in an early fall in eGFR, but is followed by marked protection against decline in kidney function, with reversal of the haemodynamic effect achieved upon drug cessation. ${ }^{12,38}$ The effect parallels that observed with RAS blockade, the only other treatment effective in slowing the progression of diabetic kidney disease, and suggests some commonality in a mechanism of action based upon reducing intraglomerular pressure - SGLT2 inhibitors by enhancing afferent arteriolar vasoconstriction and RAS blockers by increasing efferent arteriolar vasodilatation. ${ }^{39}$ While this may be the most plausible mechanistic explanation for the renoprotective effect of these agents, the effect on kidney haemodynamics has only been mechanistically studied in patients with type 1 diabetes with wholekidney hyperfiltration; whether these effects are also observed in patients with type 2 diabetes is not 
currently known. ${ }^{37}$ Other pathways by which SGLT2 inhibitors may protect the kidney an area of active study and include (but are not limited to): enhancing oxygenation of the kidney (through a reduction in tubular energy requirements), metabolic and anti-inflammatory effects, albuminuria lowering, and direct effects on glomerular endothelial function ${ }^{28,40}$

Protection against AKI is a welcome finding given early concerns about a potential increase in risk. These findings strengthen those of a previous meta-analysis of the cardiovascular outcome trials. ${ }^{16}$ While serious and non-serious AKI events were investigator reported, collected variably and not adjudicated, the large number of events and consistency of effect across the trials is striking and gives confidence to the observation that SGLT2 inhibitors provide protection against AKI. The mechanism is unknown, but could involve reduced energy expenditure in the proximal tubule, thus improving oxygenation and reducing the susceptibility of tubular cells to acute ischemic or volumerelated insults. ${ }^{28,41,42}$ Clearly any reduction in the risk of AKI with SGLT2 inhibition must be considered in the context of other adverse effects that might also occur during an acute intercurrent illness (such as ketoacidosis), and further work is needed to better understand the mechanism(s) by which SGLT2 inhibitors reduce the risk of AKI and how this evidence might be applied in practice.

The validity of these overview results is reinforced by the high quality of the included studies, but there are a number of limitations that should be considered when interpreting our findings. We included only event-driven cardiovascular or kidney outcome trials with substantial accrued followup time. This was necessary as our main interest was in assessing the need for dialysis, transplantation or death due to kidney disease, which was unlikely to be observed or responsive to therapy in trials of short duration. A substantial proportion of the data were derived from a single study of canagliflozin (CREDENCE trial) that was stopped early, which may increase the risk of overestimating treatment effects, ${ }^{43}$ however the consistency with the results from the other trials reduces that risk, and is an important finding of this review. While the consistency of effects among 
other members of the class therefore remains somewhat uncertain, there is currently no evidence of substantive heterogeneity The broader generalizability of these results may also be somewhat affected by the characteristics of participants in the included studies, and thus future work to assess the use of these agents in routine clinical practice will be important. Data on AKI might be less robust than for other endpoints, due to variances in the collection and reporting of this outcome. The effects of SGLT2 inhibition on kidney (and cardiovascular and safety) outcomes in patients with eGFR $<30 \mathrm{~mL} / \mathrm{min} / 1.73 \mathrm{~m}^{2}$ also remains an important and unanswered question, as does the comparative effectiveness of SGLT2 inhibitors against other glucose lowering agents such as glucagon-like peptide-1 (GLP-1) receptor agonists that have also demonstrated promising effects on kidney outcomes. ${ }^{44}$

Other kidney outcome trials for dapagliflozin (DAPA-CKD, NCT03036150) and empagliflozin (EMPA-KIDNEY, NCT03594110) are underway, and are expected to further enrich our understanding of the role of SGLT2 inhibitors for the prevention of kidney failure. ${ }^{24,45}$ Both trials are recruiting participants with and without T2DM on the basis of the hypothesized non-glycaemic mechanism(s) of renoprotection. Additionally the SCORED trial (NCT03315143) is testing the effects of sotagliflozin, a combined SGLT1 and SGLT2 inhibitor, on a primary cardiovascular endpoint (with other secondary kidney outcomes prespecified) in participants with T2DM and reduced kidney function. These trials will include participants with starting eGFR as low as 20 $\mathrm{mL} / \mathrm{min} / 1.73 \mathrm{~m}^{2}$ and will thus provide some important information on the effects of SGLT2 inhibition in patients with more advanced kidney disease. Future studies evaluating the combination of SGLT2 inhibitors with other glucose lowering agents that have shown beneficial impacts on kidney function, such as GLP-1 receptor agonists, are another potential area of interest, particularly in patients with established diabetic kidney disease. ${ }^{46}$ 
In conclusion, SGLT2 inhibition reduces the risk of dialysis, transplantation or death due to kidney disease, in people with T2DM and a broad range of kidney risk. These data provide substantive evidence supporting the use of SGLT2 inhibitors to prevent clinically important, patient-level kidney outcomes in individuals with T2DM. 


\section{Contributions}

BLN, VP, and MJJ contributed to the concept and design of this study. BLN contributed to the literature search, data extraction, risk of bias assessment, data analysis, interpretation, and writing of the manuscript. TY contributed to the literature search, data extraction, risk of bias assessment, interpretation, and drafting of the manuscript. LB contributed to the statistical analysis, interpretation, and critical review of the manuscript. HJLH, BN, VP, KWM, DMC, DCW, CA, SB, and AL contributed to the interpretation, writing, and critical review of the manuscript. BLN and MJJ drafted the first version of the manuscript and all authors contributed to revisions. All authors had full access to the data and take responsibility for the integrity of the data and accuracy of the data analysis.

\section{Acknowledgements}

The authors wish to acknowledge Associate Professor Stephen Wiviott, principle investigator of the DECLARE-TIMI 58 trial, as well as the investigators and sponsors of individual studies included in this systematic review and meta-analysis (Boehringer Ingelheim, Janssen, and AstraZeneca) who provided additional data and clarified outcome definitions when required. BLN is supported by an Australian National Health and Medical Research Council Postgraduate Scholarship, a University Postgraduate Award from UNSW Sydney, the Graduate Research Fund at Lincoln College and an Oxford Australia Clarendon Scholarship from the University of Oxford. BN has received research support from the Australian National Health and Medical Research Council Principal Research Fellowship. VP is receiving research support from the Australian National Health and Medical Research Council (Senior Research Fellowship and Program Grant). MJJ is supported by a Medical Research Future Fund Next Generation Clinical Researchers Program Career Development Fellowship.

\section{Declarations of interest}


BLN has received travel support from Janssen. TY has received sponsorship to attend meetings by Eli Lily and Novo Nordisk. HJLH has served as a consultant for Abbvie, Astellas, AstraZeneca, Boehringer Ingelheim, Fresenius, Janssen and Merck; and has received grant support from AstraZeneca and Boehringer Ingelheim, with all honoraria paid to his institution. BN has received research support from Janssen, Roche, Servier, and Merck Schering Plough; and is serving on advisory boards and/or has involvement in CME pro- grams for Abbott, Janssen, Novartis, Pfizer, Roche and Servier, with any consultancy, honoraria, or travel support paid to his institution. VP is serving on Steering Committees for AbbVie, Boehringer Ingelheim, GlaxoSmithKline, Janssen and Pfizer; and is serving on advisory boards and/or speaking at scientific meetings for AbbVie, Astellas, AstraZeneca, Bayer, Baxter, Bristol-Myers Squibb, Boehringer Ingelheim, Durect, Eli Lilly, Gilead, GlaxoSmithKline, Janssen, Merck, Novartis, Novo Nordisk, Pfizer, Pharmalink, Relypsa, Roche, Sanofi, Servier and Vitae. KWM reports receiving grants from Afferent, Amgen, Apple Inc., AstraZeneca, Cardiva Medical, Daiichi, Ferring, Google (Verily), Johnson \& Johnson, Luitpold, Medtronic, Merck, Novartis, Sanofi, St Jude and Tenax, receiving personal fees from Ablynx, AstraZeneca, Baim Institute, Boehringer Ingelheim, Bristol-Myers Squibb, Cardiometabolic Health Congress, Elsevier, GlaxoSmithKline, Johnson \& Johnson, Medscape, Merck, Mitsubishi, Myokardia, Novartis, Oculeve, Portola, Radiometer, Springer Publishing, Theravance, University of California San Francisco and WebMD and having equity in BioPrint Fitness. DMC has served on clinical events committees or data safety and monitoring boards for PLC Medical, AstraZeneca, Allena Pharmaceuticals, and Merck. He has served on Steering Committees for Zoll Medical and Janssen Pharmaceuticals. He has reported consulting fees or travel from Daichi Sankyo, Fresenius and Medtronic/Coviden. DCW reports having received consultancy fees from Akebia Therapeutics, Amgen, AstraZeneca, Boehringer Ingelheim, GlaxoSmithKline, Janssen, Ono, Napp, Mundipharma and Vifor Fresenius; speaker honoraria from Amgen and Vifor Fresenius; and research support from AstraZeneca. MJJ is responsible for research projects that have received unrestricted funding from Gambro, Baxter, CSL, Amgen, Eli 
Lilly and Merck; has served on advisory boards sponsored by Akebia, Baxter and Boehringer Ingelheim; has spoken at scientific meetings sponsored by Janssen, Amgen and Roche, with any consultancy, honoraria or travel support paid to her institution. LB and SB report being full-time employees of The George Institute for Global Health, the parent organization of George Clinical, a contract research organization that provides research services to Janssen for trials of SGLT2 inhibitors. CA, and AL report no relevant declarations of interest. VP, KWM, HLJH, MJJ, BN, DCW, AL, and DMC were on the steering committee for a kidney outcome trial of an SGLT2 inhibitor (canagliflozin), with VP and KWM serving as Chair and Co-Chair. 


\section{REFERENCES}

1 Liyanage $\mathrm{T}$, Ninomiya $\mathrm{T}$, Jha $\mathrm{V}$, et al. Worldwide access to treatment for end-stage kidney disease: a systematic review. Lancet 2015; 385: 1975-82.

2 Thomas MC, Cooper ME, Zimmet P. Changing epidemiology of type 2 diabetes mellitus and associated chronic kidney disease. Nat Rev Nephrol 2016; 12: 73-81.

3 Neuen BL, Chadban SJ, Demaio AR, Johnson DW, Perkovic V. Chronic kidney disease and the global NCDs agenda. BMJ Global Health 2017; e000380.

4 Lewis EJ, Hunsicker LG, Bain RP, Rohde RD. The effect of angiotensin-converting-enzyme inhibition on diabetic nephropathy. N Engl J Med 1993; 329: 1456-62.

5 Lewis EJ, Hunsicker LG, Clarke WR, et al. Renoprotective effect of the angiotensin-receptor antagonist irbesartan in patients with nephropathy due to type 2 diabetes. $N$ Engl J Med 2001; 345: 851-60.

6 Brenner BM, Cooper ME, de Zeeuw D, et al. Effects of losartan on renal and cardiovascular outcomes in patients with type 2 diabetes and nephropathy. N Engl J Med 2001; 345: 861-9.

7 Gross JL, De Azevedo MJ, Silveiro SP, Canani LH, Caramori ML, Zelmanovitz T. Diabetic nephropathy: diagnosis, prevention, and treatment. Diabetes Care 2005; 28: 164-76.

8 Webster AC, Nagler EV, Morton RL, Masson P. Chronic kidney disease. Lancet 2017; 389: $1238-52$.

9 Heerspink HJ, Perkins BA, Fitchett DH, Husain M, Cherney DZ. Sodium glucose cotransporter 2 inhibitors in the treatment of diabetes: cardiovascular and kidney effects, potential mechanisms and clinical applications. Circulation 2016; 134: 752-72.

10 Perkovic V, de Zeeuw D, Mahaffey KW, et al. Canagliflozin and renal outcomes in type 2 diabetes: results from the CANVAS Program randomised clinical trials. Lancet Diabetes Endocrinol 2018; 6: 691-704.

11 Wanner C, Inzucchi SE, Lachin JM, et al. Empagliflozin and progression of kidney disease in type 2 diabetes. $N$ Engl J Med 2016; 375: 323-34.

12 Neuen BL, Ohkuma T, Neal B, et al. Cardiovascular and Renal Outcomes With Canagliflozin According to Baseline Kidney Function: Data from the CANVAS Program. Circulation 2018; 138: $1537-50$.

13 Wanner C, Lachin JM, Inzucchi SE, et al. Empagliflozin and clinical outcomes in patients with type 2 diabetes, established cardiovascular disease and chronic kidney disease. Circulation 2017; 137: 119-29.

14 Zelniker TA, Wiviott SD, Raz I, et al. SGLT2 inhibitors for primary and secondary prevention of cardiovascular and renal outcomes in type 2 diabetes: a systematic review and meta-analysis of cardiovascular outcome trials. Lancet 2018; 393: 31-9.

15 Mosenzon O, Wiviott SD, Cahn A, et al. Effects of dapagliflozin on development and progression of kidney disease in patients with type 2 diabetes: an analysis from the DECLARE-TIMI 58 randomised trial. Lancet Diabetes Endocrinol 2019; published online June. DOI:10.1016/S2213-8587(19)30180-9. 
16 Gilbert RE, Thorpe KE. Acute Kidney Injury with sodium-glucose linked cotransporter-2 inhibitors: a meta-analysis of cardiovascular outcome trials. Diabetes Obes Metab 2019. DOI:doi: 10.1111/dom.13754.

17 Perkovic V, Jardine MJ, Neal B, et al. Canagliflozin and Renal Outcomes in Type 2 Diabetes and Nephropathy. N Engl J Med 2019.

18 Higgins JP, Altman DG, Gøtzsche PC, et al. The Cochrane Collaboration's tool for assessing risk of bias in randomised trials. BMJ 2011; 343: d5928.

19 Wanner C, Inzucchi SE, Zinman B. Empagliflozin and progression of kidney disease in type 2 diabetes. N Engl J Med 2016; 375: 1801-2.

20 Neal B, Perkovic V, Mahaffey KW, et al. Optimizing the analysis strategy for the CANVAS Program: A prespecified plan for the integrated analyses of the CANVAS and CANVAS-R trials. Diabetes Obes Metab 2017; 19: 926-35.

21 Perkovic V, de Zeeuw D, Mahaffey KW, et al. Canagliflozin and renal outcomes in type 2 diabetes: results from the CANVAS Program randomised clinical trials. Lancet Diabetes Endocrinol 2018; 6: 691-704.

22 Wanner C, Heerspink HJ, Zinman B, et al. Empagliflozin and kidney function decline in patients with type 2 diabetes: a slope analysis from the EMPA-REG outcome trial. J Am Soc Nephrol 2018; 29: 2755-69.

23 Mayer GJ, Wanner C, Weir MR, et al. Analysis from the EMPA-REG OUTCOME((R)) trial indicates empagliflozin may assist in preventing the progression of chronic kidney disease in patients with type 2 diabetes irrespective of medications that alter intrarenal hemodynamics. Kidney Int 2019; published online March. DOI:10.1016/j.kint.2019.02.033.

24 Herrington WG, Preiss D, Haynes R, et al. The potential for improving cardio-renal outcomes by sodium-glucose co-transporter-2 inhibition in people with chronic kidney disease: a rationale for the EMPA-KIDNEY study. Clin Kidney J 2018; 11: 749-61.

25 Neal B, Perkovic V, Mahaffey KW, et al. Canagliflozin and cardiovascular and renal events in type 2 diabetes. N Engl J Med 2017; 377: 644-57.

26 Zinman B, Wanner C, Lachin JM, et al. Empagliflozin, cardiovascular outcomes, and mortality in type 2 diabetes. $N$ Engl J Med 2015; 373: 2117-28.

27 Wiviott SD, Raz I, Bonaca MP, et al. Dapagliflozin and cardiovascular outcomes in type 2 diabetes. N Engl J Med 2018; 380: 347-57.

28 Heerspink HJ, Kosiborod M, Inzucchi SE, Cherney DZ. Renoprotective effects of sodiumglucose cotransporter-2 inhibitors. Kidney Int 2018; 94: 26-39.

29 Toyama T, Neuen BL, Jun M, et al. Effect of SGLT2 inhibitors on cardiovascular, renal and safety outcomes in patients with type 2 diabetes mellitus and chronic kidney disease: a systematic review and meta-analysis. Diabetes Obes Metab 2019; 21: 1237-50.

30 Gansevoort RT, Matsushita K, Van Der Velde M, et al. Lower estimated GFR and higher albuminuria are associated with adverse kidney outcomes. A collaborative meta-analysis of general and high-risk population cohorts. Kidney Int 2011; 80: 93-104. 
31 Jafar TH, Schmid CH, Landa M, et al. Angiotensin-converting enzyme inhibitors and progression of nondiabetic renal disease: a meta-analysis of patient-level data. Ann Intern Med 2001; 135: 73-87.

32 Kent DM, Jafar TH, Hayward RA, et al. Progression risk, urinary protein excretion, and treatment effects of angiotensin-converting enzyme inhibitors in nondiabetic kidney disease. $J$ Am Soc Nephrol 2007; 18: 1959-65.

33 Molitch ME, Adler AI, Flyvbjerg A, et al. Diabetic kidney disease: a clinical update from Kidney Disease: Improving Global Outcomes. Kidney Int 2015; 87: 20-30.

34 Cherney DZ, Zinman B, Inzucchi SE, et al. Effects of empagliflozin on the urinary albuminto-creatinine ratio in patients with type 2 diabetes and established cardiovascular disease: an exploratory analysis from the EMPA-REG OUTCOME randomised, placebo-controlled trial. Lancet Diabetes Endocrinol 2017; 5: 610-21.

35 Alicic RZ, Rooney MT, Tuttle KR. Diabetic Kidney Disease Challenges, Progress, and Possibilities. Clin J Am Soc Nephrol 2017; 12: 2032-45.

36 Tonneijck L, Muskiet MH, Smits MM, et al. Glomerular hyperfiltration in diabetes: mechanisms, clinical significance, and treatment. J Am Soc Nephrol 2017; 28: 1023-39.

37 Cherney DZ, Perkins BA, Soleymanlou N, et al. Renal hemodynamic effect of sodium-glucose cotransporter 2 inhibition in patients with type 1 diabetes mellitus. Circulation 2014; 129: 587-97.

38 Fioretto P, Del Prato S, Buse JB, et al. Efficacy and Safety of Dapagliflozin in Patients with Type 2 Diabetes and Moderate Renal Impairment (Chronic Kidney Disease Stage 3A): The DERIVE Study. Diabetes Obes Metab 2018; 20: 2532-40.

39 Tuttle KR. Back to the future: Glomerular hyperfiltration and the diabetic kidney. Diabetes 2017; 66: 14-6.

40 Heerspink HJ. Sodium glucose co-transporter 2 inhibition: a new avenue to protect the kidney. Nephrol Dial Transplant 2019; DOI: 10.1093/ndt/gfz033

41 Chang Y-K, Choi H, Jeong JY, et al. Dapagliflozin, SGLT2 inhibitor, attenuates renal ischemia-reperfusion injury. PLoS One 2016; 11: e0158810.

42 O'Neill J, Fasching A, Pihl L, Patinha D, Franzén S, Palm F. Acute SGLT inhibition normalizes $\mathrm{O} 2$ tension in the renal cortex but causes hypoxia in the renal medulla in anaesthetized control and diabetic rats. Am J Physiol Renal Physiol 2015; 309: F227-34.

43 Viele K, McGlothlin A, Broglio K. Interpretation of clinical trials that stopped early. JAMA 2016; 15: 1646-7.

44 Zelniker TA, Wiviott SD, Raz I, et al. Comparison of the Effects of Glucagon-Like Peptide Receptor Agonists and Sodium-Glucose Co-Transporter 2 Inhibitors for Prevention of Major Adverse Cardiovascular and Renal Outcomes in Type 2 Diabetes Mellitus: A Systematic Review and Meta-Analysis of Cardiovascular Outcomes Trials. Circulation 2019; 139: 202231.

45 Pecoits-Filho R, Perkovic V. Are SGLT2 Inhibitors Ready for Prime Time for CKD? Clin J 
Am Soc Nephrol 2017; 13: 318-20.

46 Goncalves E, Bell DS. Combination treatment of SGLT2 inhibitors and GLP-1 receptor agonists: symbiotic effects on metabolism and cardiorenal risk. Diabetes Ther 2018; 9: 91926. 


\section{FIGURE LEGENDS}

Figure 1. Effect of SGLT2 inhibitors on dialysis, transplantation or death due to kidney disease.

SGLT2: sodium-glucose cotransporter 2 inhibitor; RR: relative risk; CI: confidence interval.

Figure 2. Effect of SGLT2 inhibitors on (A) ESKD, (B) substantial loss of kidney function, ESKD, or death due to kidney disease and (C) substantial loss of kidney function, ESKD, or death due to cardiovascular or kidney disease.

ESKD: end-stage kidney disease; SGLT2: sodium-glucose cotransporter 2 inhibitor; RR: relative risk; CI: confidence interval.

Figure 3. Effect of SGLT2 inhibitors on substantial loss of kidney function, ESKD or death due to kidney disease, stratified by baseline (A) eGFR, (B) UACR, and (C) use of reninangiotensin system blockade.

ESKD: end-stage kidney disease; eGFR: estimated glomerular filtration rate; UACR: urinary albumin:creatinine ratio; SGLT2: sodium-glucose cotransporter 2 inhibitor; RR: relative risk; CI: confidence interval.

Figure 4. Effect of SGLT2 inhibitors on acute kidney injury.

SGLT2: sodium-glucose cotransporter 2 inhibitor; RR: relative risk; CI: confidence interval.

Figure 5. Summary of the effects of SGLT2 inhibition on major kidney outcomes

SGLT2: sodium-glucose cotransporter 2 inhibitor; RR: relative risk; CI: confidence interval 
SGLT2 inhibitors for the prevention of kidney failure in patients with type 2 diabetes: a systematic review and meta-analysis

Brendon L. Neuen MBBS(Hons) ${ }^{1}$, Tamara Young MBBS(Hons) ${ }^{1}$, Prof Hiddo J.L. Heerspink $\mathrm{PhD}^{1,2}$, Prof Bruce Neal PhD ${ }^{1,3,4}$, Prof Vlado Perkovic $\mathrm{PhD}^{1}$, Laurent Billot MSc ${ }^{1}$, Prof Kenneth W. Mahaffey MD ${ }^{5}$, David M. Charytan $\mathrm{MD}^{6}$, Prof David C. Wheeler MD, ${ }^{7}$ Clare Arnott $\mathrm{PhD}^{1,3,8}$, Formatted: Not Superscript/ Subscript Severine Bompoint $\mathrm{BSc}^{1}$, Prof Adeera Levin $\mathrm{MD}^{9}$, Meg J. Jardine $\mathrm{PhD}^{1,10}$

${ }^{1}$ The George Institute for Global Health, UNSW Sydney, Australia; ${ }^{2}$ University of Groningen, University Medical Center Groningen, Groningen, The Netherlands; ${ }^{3}$ The Charles Perkins Centre, University of Sydney, Sydney, Australia; ${ }^{4}$ Epidemiology and Biostatistics, Imperial College London, London, UK; ${ }^{5}$ Stanford Center for Clinical Research, Department of Medicine, ${ }^{5}$ Stanford University School of Medicine, Stanford University, Stanford, USA; ${ }^{6}$ Nephrology Division, Department of Medicine, NYU Langone Medical Center, New York, USA; ${ }^{7}$ Department of Renal Medicine, University College London, London, United Kingdom, ${ }^{8}$ Department of Cardiology, Royal Prince Alfred Hospital, Sydney, Australia; ${ }^{9}$ Division of Nephrology, University of British Columbia, Vancouver, Canada; ${ }^{10}$ Concord Repatriation and General Hospital, Sydney, Australia

Manuscript type: Systematic review and meta-analysis

Word count: $\underline{431646}$

Abstract word count: $3 \underline{61} \theta \theta$

Running title: SGLT2 inhibitors and kidney failure

Keywords: SGLT2 inhibitors, type 2 diabetes, renal outcomes, systematic review, meta-analysis

Corresponding author: Associate Professor Meg Jardine

Address: Level 5, 1 King Street Newtown NSW 2042 Australia

Phone: +6128052 4300 Fax: +61280524301

Email: mjardine@georgeinstitute.org.au 


\section{RESEARCH IN CONEXT}

\section{Evidence before this study}

Large-scale randomized cardiovascular outcome trials of sodium glucose cotransporter-2 (SGLT2) inhibitors have suggested promising effects on albuminuria and creatinine based kidney outcomes. However, these trials included few participants at high risk of clinically important kidney outcomes, and as a result, the effect of SGLT2 inhibitors on kidneythe outcomes of greatest concern to patients - namely, the need for long-term dialysis, transplantation or death due to kidney disease - has been uncertain. SGLT2 inhibitors are also not currently approved for use in patients with estimated glomerular filtration rate (eGFR) $<45$ or $60 \mathrm{~mL} / \mathrm{min} / 1.73 \mathrm{~m}^{2}$ in most countries, primarily because their glucose lowering effect is substantially dependent on kidney function. A recent meta-analysis of these trials has reported that the renoprotective effect of SGLT2 attenuates with declining kidney function. However, less than a sixth of participants studied had a baseline eGFR less than 60 $\mathrm{mL} / \mathrm{min} / 1 \cdot 73 \mathrm{~m}^{2}$, and even fewer had baseline eGFR $<45 \mathrm{~mL} / \mathrm{min} / 1 \cdot 73 \mathrm{~m}^{2}$. The ability to robustly assess effects in people with reduced kidney function was therefore limited, especially since few patient-level kidney outcomes occurred. While collectively these trials have suggested that SGLT2 inhibitors might protect against acute kidney injury, the safety of these agents in patients at high risk of adverse kidney outcomes has remained a concern. More recently the CREDENCE trial (Canagliflozin and Renal Endpoints in Diabetes with Established Nephropathy Clinical Evaluation) has been published, which was designed specifically to examine the impact of SGLT2 inhibition in people at high risk of kidney disease progression. These agents are also not currently approved for use in patients with estimated glomerular filtration rate (eGFR) less than 45 or $60 \mathrm{~mL} / \mathrm{min} / 1.73 \mathrm{~m}^{2}$ in most countries, primarily because their glucose lowering effect is dependent on kidney function. We therefore conducted a systematic review and meta-analysis of randomized, controlled, eventdriven, cardiovascular and kidney outcome trials reporting effects of major kidney outcomes in patients with type 2 diabetes. MEDLINE and EMBASE were searched from inception until Mareh 4June 142019 to identify potentially relevant studies. The protocol for this systematic review and 
meta-analysis was prospectively-registered in the International Prospective Register of Systematic

Reviews before the analyses were completed (PROSPERO registration number CRD42019131774).

\section{Added value of this study}

This review summarises data from four studies including 38,723 participants across six continents.

There was clear evidence that SGLT2 inhibitors reduce the risk dialysis, transplantation or death due to kidney disease, as well as a range of other major kidney outcomes, and that these agents also provide protection against acute kidney injury. Additionally, there were definitive, separate benefits at all levels of kidney function, including an approximate $30 \%$ proportional risk reduction in the composite kidney outcome in participants with baseline eGFR less than $45 \mathrm{~mL} / \mathrm{min} / 1 \cdot 73 \mathrm{~m}^{2}$ in whom these drugs are mostly not permitted for use.

\section{Implications of all the available evidence}

These results provide the strongest evidence yet that SGLT2 inhibitors should be routinely offered to individuals with type 2 diabetes at risk of progressive kidney disease. The clear evidence of renoprotection across the spectrum of kidney function studied to date call into question current restrictions on the use of SGLT2 inhibitors in people with reduced kidney function, and suggest that many more individuals with type 2 diabetes at high risk of kidney failure are likely to benefit from treatment. 


\section{SUMMARY}

\section{Background}

The impact effect of sodium-glucose cotransporter 2 (SGLT2) inhibitors on kidney failure, particularly the need for dialysis and transplantation, or death due to kidney disease, has been uncertain. Previous studies have also been underpowered to robustly assess heterogeneity of effects on kidney outcomes by different levels of estimated glomerular filtration rate (eGFR) and albuminuria.

\section{Methods}

We conducted a systematic review and meta-analysis of randomized, controlled, cardiovascular or kidney outcome trials of SGLT2 inhibitors reporting effects on kidney failure and other-major kidney outcomes in people with type 2 diabetes (PROSPERO registration number CRD42019131774). MEDLINE and EMBASE were searched from inception to 1 March14 June 2019 to identify eligible trials. The primary outcome was kidney failure, defined as dialysis, transplantation, dialysis, transplantation, or death due to kidney disease. We used random effects models to obtain summary relative risks (RR) with $95 \%$ confidence intervals (CI) and random effects meta-regression to explore effect modification by subgroups of baseline estimated glomerular filtration rate (eGFR), albuminuria, and use of renin-angiotensin system (RAS) blockade.

\section{Findings}

Data were obtained from four studies including 38,723 participants of whom 25218 reached kidney failurerequired dialysis or transplantation or died due to kidney disease, 3 3510 developed end-stage kidney disease (ESKD), and 943 experienced acute kidney injury (AKI). SGLT2 inhibitors reduced the risk of kidney failuredialysis, transplantation or death due to kidney disease by $29 \%$ (RR

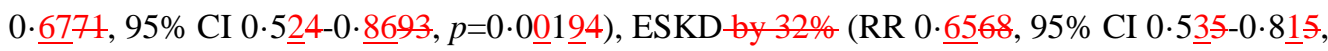
$p \leq \equiv 0 \cdot \underline{0001})$, and AKI by $25 \%(\operatorname{RR} 0 \cdot 75,95 \%$ CI 0.66-0 85, $p<0 \cdot 0001)$, with consistent benefits across studies. While there was some evidence that the proportional effect of SGLT2 inhibitors 
might attenuate with declining kidney function $(P$-trend $=0 \cdot 07 \underline{3})$, there was clear, separate evidence of benefit for all eGFR subgroups, including for participants with baseline-starting eGFR $\underline{30-<45}$ $\mathrm{mL} / \mathrm{min} / 1 \cdot 73 \mathrm{~m}^{2}(\mathrm{RR} 0 \cdot 70,95 \%$ CI $0 \cdot 54-0 \cdot 91, p=0 \cdot 0080)$. Renoprotection was also consistent irrespective of baseline albuminuria and use of RAS blockade $(P$-trend $=0.66$ and $P$ -

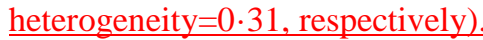

\section{Interpretation}

SGLT2 inhibitors reduce the risk of kidney failuredialysis, transplant or death due to kidney disease and provide protection against acute kidney injury. These data provide substantive evidence supporting the use of SGLT2 inhibitors to prevent major kidney outcomes in people with type 2 diabetes.

\section{Funding}

None. 


\section{INTRODUCTION}

It is estimated that approximately 2.6 million people received dialysis or underwent kidney transplantation for kidney failure in 2010, and this number is projected to more than double by 2030. ${ }^{1}$ In many countries, more than half of all patients entering dialysis programmes have type 2 diabetes mellitus (T2DM), making it a leading cause of kidney failure worldwide. ${ }^{2}$ Kidney failure due to T2DM is a large and growing challenge, not only for patients and their families and caregivers, but also for health systems and governments. . $^{2}$

Treatment with angiotensin converting enzyme (ACE) inhibitors or angiotensin-receptor blockers (ARBs) has been shown to prevent major kidney outcomes in people with diabetes, and these agents are currently recommended by clinical practice guidelines for the treatment of people with T2DM who have, or are at high risk of, kidney disease. $\frac{4-8}{}$ However, the residual risk remains high with new treatments urgently needed to reduce the growing burden of kidney failure.

SGLT2 inhibitors are a newer class of glucose-lowering agent that also lower blood pressure, body weight and albuminuria, and may have direct haemodynamic effects on the kidney. ${ }^{9}$ Large scale randomized trials of SGLT2 inhibitors, which were originally designed to meet regulatory requirements and ensure cardiovascular safety, have demonstrated promising effects on a range of albuminuria and serum creatinine based kidney outcomes in patients with, or at high risk of, atherosclerotic cardiovascular disease. ${ }^{10-15}$ These multiple effects have translated into a reduction in eardiovascular events in people with T2DM in large cardiovascular outcome trials. ${ }^{10-12}$ The trials also demenstrated promising effects on a range of albuminuria and serum creatinine based kidney euteomes. ${ }^{13-16}$-The majority of participants in these trials were at low risk of clinically important kidney outcomes, and as a result, event rates for kidney failure were low, with few participants requiring dialysis or kidney transplantation, or dying due to kidney disease, in each trial. As they were also not explicitly designed to provide definitive information on renoprotective effects, kidney 
endpoints were not always pre-specified, were not always adjudicated, and the distinction between acute and chronic reductions in estimated glomerular filtration rate (eGFR) was not possible in all studies.

A recent meta-analysis of cardiovascular outcome trials suggested that the effect of SGLT2 inhibitors on kidney outcomes attenuates with declining eGFR. ${ }^{14}$ However, less than a sixth of participants in this analysis had baseline eGFR $<60 \mathrm{~mL} / \mathrm{min} / 1.73 \mathrm{~m}^{2}$, and thus the ability to robustly assess effect modification by kidney function has been limited. Additionally, because very few participants with starting eGFR $<45 \mathrm{~mL} / \mathrm{min} / 1 \cdot 73 \mathrm{~m}^{2}$ have been studied, it has also been unclear whether these patients derive protection against kidney outcomes, since the glycaemic efficacy of these agents is substantially attenuated in this population. Similarly, few participants with higher levels of albuminuria have been studied, and therefore the consistency of treatment effect across different levels of albuminuria is unclear. While these trials have collectively suggested protection against acute kidney injury, ${ }^{16}$ the safety of these agents in patients at higher risk of adverse kidney outcomes has not been well established. Furthermore, since each trial included only modest numbers of patients at any given level of kidney function or albuminuria, the ability to robustly examine the consistency of treatment effect across different levels of kidney function or albuminuria has been limited.Most recently, the Canagliflozin and Renal Endpoints in Diabetes with Established Nephropathy Clinical Evaluation (CREDENCE) trial has been reported, which is the first study designed to specifically evaluate the impact of an SGLT2 inhibitor on a primary kidney outcome in people with established diabetic kidney disease. ${ }^{17}$

We therefore undertook a systematic review and meta-analysis to determine the consistency of effect size across SGLT2 trials and different levels of kidney function and albuminuria, summarize results, and integrate available data on the effects of SGLT2 inhibition on the risk of clinically important kidney outcomes in people with T2DM. 


\section{METHODS}

This systematic review and meta-analysis was conducted and reported using the Preferred Reporting Items for Systematic Reviews and Meta-Analysis (PRISMA) statement. The protocol for this review was submitted before the analyses were begun (10 April 2019) and was prospectively registered in the International Prospective Register of Systematic Reviews before the analyses were completed (PROSPERO registration number CRD42019131774) and can be accessed at: http://www.crd.york.ac.uk/PROSPERO/display_record.php?ID=CRD42019131774.

\section{Search strategy and selection criteria}

We searched MEDLINE and EMBASE from inception to 1 March14 June 2019 to identify potentially eligible studies. Details of the search strategy, including text words and medical subject headings are provided in Table S1. We included all randomized, controlled, event-driven, cardiovascular or kidney outcome trials of SGLT2 inhibitors versus active or placebo control, in order to capture those with meaningful numbers of clinical kidney outcomes. Trials with extension periods and those including participants with type 1 diabetes or individuals less than 18 years of age were excluded. Two authors (B.L.N and T.Y) independently screened the titles and abstracts of all identified articles and, when required, reviewed full-text manuscripts to identify potentially relevant studies. Any disagreements related to the identification or eligibility of studies was resolved through discussion with a third author (M.J.J). Study sponsor and investigators were contacted to obtain additional trial-level data and clarify outcome definitions when required.

\section{Data synthesis and analysis}

Two authors (B.L.N and T.Y) independently extracted all data using a standardized data form and assessed risk of bias at the study level using the Cochrane Risk of Bias Tool. ${ }^{18}$ We used image extraction software to extract data presented only in figures without corresponding numerical data 
(WebPlotDigitizer version 4·1, Ankit Rohatgi, Austin, TX, https://automeris.io/WebPlotDigitizer/).

These data were summarized descriptively and not used for quantitative synthesis. Any discrepancies in data extraction or risk of bias assessment were resolved in consultation with a third author (M.J.J). Due to the small number of eligible trials, publication bias was not assessed.

The primary outcome of interest was, defined as the need for chronic dialysis, ${ }_{2}$-r kidney transplantation, or death due to kidney disease. Other kidney outcomes included: (1) ESKD, defined as chronic dialysis, kidney transplantation, or sustained eGFR less than $15 \mathrm{~mL} / \mathrm{min} / 1 \cdot 73 \mathrm{~m}^{2}$, (2) substantial loss of kidney function, ESKD, or death due to kidney disease, (3) substantial loss of kidney function, ESKD, or death due to cardiovascular or kidney disease, (4) long-term eGFR slope, and (5) acute kidney injury (AKI). Substantial loss of kidney function was preferentially defined as a sustained doubling of serum creatinine (representing an approximate 57\% decline in kidney function). Where sustained doubling of serum creatinine was not reported, we included sustained $40 \%$ decline in eGFR or unsustained doubling of serum creatinine as defined by study authors. We preferentially used data on sustained kidney outcomes confirmed with repeat assessment where these were reported to exclude acute changes in kidney function and initiation of dialysis for AKI, but accepted unsustained outcomes where these were the only ones reported. This was the case for the EMPA-REG OUTCOME trial, where sustained kidney outcome data were reported in a separate correspondence ${ }^{19}$ following the main trial publication. ${ }^{11}$ The definitions of long-term eGFR slope (reperted as the-annualized difference in eGFR between treatment and control) and AKI varied across studies and we used these outcomes as defined and reported in each study.

We prespecified that quantitatively synthesized-results for dichotomous outcomes were to be quantitatively synthesized by individual studies using a random effects model with inverse variance weighting to obtain summary effect estimates represented as relative risk (RR) with associated $95 \%$ 
confidence intervals (CI). We also decided a priori to pooled, in order of preference, hazard ratios, incidence rate ratios (events/patient-years), and risk ratios (events/number of participants) to maximize the use of trial level data from included studies, particularly for canagliflozin, where the integrated analysis and reporting of two parallel companion trials with different randomization ratios and different follow-up durations precluded the use of risk ratios. ${ }^{20}$ When studies did not report the preferred outcome definition for substantial loss of kidney function, we performed sensitivity tests excluding those studies to assess the impact of endpoint definition on the results.

We prospectively decided to summarize the effect of SGLT2 inhibitors on long-term eGFR slope descriptively bBecause of variations in the definition of this outcome and because the eGFR slope eutcomeit measured the absolute rather than proportional effect of treatment. Any; heterogeneity between studies for this outcome therefore could notcould not be meaningfully assessed, as differences in absolute effect reflected differences in baseline kidney risk. These datawere therefore summarized descriptively.For all other outcomes we assessed heterogeneity between studies using the $\mathrm{I}^{2}$ test and $P$-heterogeneity values obtained from a random effects model. $\mathrm{I}^{2}$ values of $<25 \%, 25-75 \%$, and $>75-100 \%$ were considered to reflect low, moderate, and high likelihood of differences between studies, respectively.

Because of the kidney-based mechanism of action and albuminuria lowering effect of SGLT2 inhibitors, we performed additionaprospectively decided to conduct $\underline{\text { subgroup analysees for }}$ efficacy outcomes to assess whether treatment effects varied-to assess for effect modification by three kidney-related subgroups. The effect of SGLT2 inhibitors on the composite kidney outcome of substantial loss of kidney function, ESKD or death due to kidney disease was assessed across different levels of baseline kidney function and urinary albumin excretion. Additionally, because ACE inhibitors and ARBs are recommended for the treatment of diabetic kidney disease, we also analysed whether the effects of SGLT2 inhibition differed by baseline use of renin-angiotensin system (RAS) blockade. We prospectively decided to conduct these analyses for efficacy outcomes 
(i.e. all outcomes except AKI) where subgroup data were reported in two or more studies. Because the results come from relatively few individual studies, we performed multiple sensitivity analyses to assess the vulnerability of the eGFR subgroup analysis outcomes to definitional and methodological decisions. For the outcome substantial loss of kidney function, ESKD or death due to kidney disease, w $\underline{\mathrm{W} e}$ assessed the impact of SGLT2 inhibitors by eGFR categories (eGFR <45, $45-<60,60-<90$ and $\geq 90 \mathrm{~mL} / \mathrm{min} / 1 \cdot 73 \mathrm{~m}^{2}$ ) and levels of albuminuria (urinary albumin:creatinine ratio $[\mathrm{UACR}]<30,30-300$, and $>300 \mathrm{mg} / \mathrm{g}$ ) as the main analysis. Where studies reported the eGFR $<60 \mathrm{~mL} / \mathrm{min} / 1 \cdot 73 \mathrm{~m}^{2}$ subgroup without more granular categories, as occurred in the DECLARETIMI 58 trial, we excluded these data from the main analysis. However we performed a sensitivity analysis in which the outcomes for the baseline eGFR $<60 \mathrm{~mL} / \mathrm{min} / 1 \cdot 73 \mathrm{~m}^{2}$ subgroup were included with the eGFR $45-<60 \mathrm{~mL} / \mathrm{min} / 1 \cdot 73 \mathrm{~m}^{2}$ category, on the assumption that a large majority of these participants were likely to have an eGFR $45-60 \mathrm{~mL} / \mathrm{min} / 1.73 \mathrm{~m}^{2}$ based on the trial exclusion criteria. We conducted an additional sensitivity analysis to assess the effects of treatment in participants with eGFR $<60$ and $\geq 60 \mathrm{~mL} / \mathrm{min} / 1 \cdot 73 \mathrm{~m}^{2}$. When required, effect estimates for subgroups within the same study (e.g. eGFR $30-<45$ and $45-<60 \mathrm{~mL} / \mathrm{min} / 1 \cdot 73 \mathrm{~m}^{2}$ ) were merged using a fixed effects model. For the eGFR slope outcome, data were stratified by kidney function $\left(\mathrm{eGFR}<60\right.$ and $\geq 60 \mathrm{~mL} / \mathrm{min} / 1 \cdot 73 \mathrm{~m}^{2}$ ) and albuminuria (UACR $<30,30-300$, and $>300 \mathrm{mg} / \mathrm{g}$ ) and summarized descriptively.

We decided a priori to used random effects meta-regression with restricted maximum likelihøod and Hartung Knapp adjustment to assess trend in treatment effects across eGFR and albuminuria subgroups as the primary analysis. In sensitivity analyses we repeated the meta-regression analyses treating subgroups as categories without assumptions of linearity. A $P$-trend and $P$-heterogeneity of $<0 \cdot 10$ were considered to reflect a high likelihood of differences beyond chance. 
All analyses were performed using Stata 15.1 (StataCorp 2017. Stata Statistical Software: Release

15. College Station, TX; StataCorp LLC).

\section{Role of the funding source}

This study was not specifically funded. All authors had full access to the data and agreed on the final decision to submit for publication.

\section{RESULTS}

We identified four separate studies comprising five individual trials after applying the search strategy and study selection criteria (Table S1 and Figure S1). Data from a number of secondary analyses of these studies were used in this meta-analysis. ${ }^{11-13,15,19,21-24}$ One secondary analysis of the DECLARE-TIMI 58 trial which was published after the systematic literature search was identified by hand searching. ${ }^{15}$ The CANVAS Program comprised two companion trials (CANVAS and CANVAS-R) that were conducted in parallel and analysed and reported as a single program. $\stackrel{25}{\text { All }}$ studies compared an SGLT2 inhibitor with matching placebo. Three studies were designed as cardiovascular outcome trials testing the impact of empagliflozin, canagliflozin, and dapagliflozin on a primary composite cardiovascular outcome of nonfatal myocardial infarction, nonfatal stroke, or cardiovascular death, with a range of pre-specified exploratory and post-hoc kidney outcomes also reported. $\frac{25-27}{}$ One study was an event-driven kidney outcome trial for canagliflozin with a primary composite outcome of sustained doubling of serum creatinine, ESKD, or death due to cardiovascular or kidney disease. ${ }^{17}$ The risk of bias was low for all indicators; all participants and investigators were blinded to treatment allocation with complete reporting of outcomes (Figure S2).

In total, this meta-analysis included data on 38,723 randomised participants from six continents. The mean age across the studies ranged from $61 \cdot 3$ to 63.9 years, while $35.0 \%$ of participants overall were female (Table 1). The proportion of participants with eGFR $<60 \mathrm{~mL} / \mathrm{min} / 1 \cdot 73 \mathrm{~m}^{2}$ 
ranged from $7.4 \%$ in DECLARE-TIMI 58 to $58.98 \%$ in CREDENCE. The majority of participants in the three cardiovascular outcome trials had a UACR $<30 \mathrm{mg} / \mathrm{g}$ at baseline (range $59.4 \%$ to 69.18\%), while $88.0 \%$ in CREDENCE had a baseline UACR $>300 \mathrm{mg} / \mathrm{g}$ was an entry criterion for the CREDENCE trial (Table 1). An eGFR of $\geq 30 \mathrm{~mL} / \mathrm{min} / 1 \cdot 73 \mathrm{~m}^{2}$ was an inclusion criterion in all studies with the exception of the DECLARE-TIMI 58 trial where Cockroft Gault creatinine clearance of $\geq 60 \mathrm{~mL} / \mathrm{min}$ was required. Treatment with RAS blockade was required for entry by the CREDENCE trial only. Accordingly, virtually all (99.9\%) CREDENCE participants were treated with ACE inhibitors or ARBs at baseline compared with approximately $80 \%$ of participants in the other trials (Table 1).

Overall, there were 218-252 eccurrences of kidney failureparticipants who required dialysis or transplantation or died due to kidney disease. There were 33510 ESKD events; 967 incidences of substantial loss of kidney function, ESKD or death due to kidney disease; 2,323 cases of substantial loss of kidney function, ESKD, or death due to cardiovascular or kidney disease; and 943 episodes of AKI. Pre-specification of outcomes, requirements for changes in kidney function to be confirmed on repeated measurement, and adjudication procedures differed across the studies (Table S3). Kidney endpoints were also defined and reported variably across the studies (Table S4-S5).

Primary outcome

SGLT2 inhibitors reduced the risk of kidney failuredialysis, transplantation or death due to kidney disease by $29 \underline{33} \%$ (RR 0.6771, 95\% CI 0.524-0. $\underline{69} 93, p=0 \cdot 0 \underline{0194}$ ). The effect of SGLT2 inhibitors on this outcome was consistent across studies $\left(\mathrm{I}^{2}=0 \%, P\right.$-heterogeneity $=0 \cdot 79 \underline{53}$; Figure 1$)$.

\section{Other kidney outeomes}

SGLT2 inhibitors reduced the risk of ESKD by $3 \underline{5} z \%$ (RR $0 \cdot 6 \underline{5} 8,95 \%$ CI $0.5 \underline{3} 5-0 \cdot 8 \underline{1}$, $p \leq=0 \cdot \underline{0001})$, with no differences in treatment effect across studies $\left(\mathrm{I}^{2}=0 \cdot 0 \%, P\right.$ - 
heterogeneity=0.4195). SGLT2 inhibition also reduced the risk of substantial loss of kidney

function, ESKD, or death due to kidney disease by $42 \%(0 \cdot 58,95 \%$ CI $0 \cdot 51-0 \cdot 66, p<0 \cdot 0001$;

Figure 2) with no evidence of differences between studies $\left(\mathrm{I}^{2}=0 \%, P\right.$-heterogeneity $\left.=0 \cdot 49\right)$. The results from sensitivity testing which excluded studies that did not report our preferred event definition of substantial loss of kidney function were essentially unchanged (Table S6). The overall effect of SGLT2 inhibitors on substantial loss of kidney function, ESKD, death due to cardiovascular or kidney disease (RR $0 \cdot 71,95 \%$ CI 0.0.63-0.82, $p<0 \cdot 0001$; Figure 2) varied across studies, primarily due to the EMPA-REG OUTCOME trial $\left(\mathrm{I}^{2}=60 \cdot 3 \%, P\right.$-heterogeneity=0.056).

SGLT2 inhibitors also lowered the risk of AKI by $25 \%$ (RR 0.75, 95\% CI 0.66-0.85, $p<0 \cdot 0001$; Figure 4), with no evidence of differences between studies $\left(\mathrm{I}^{2}=0 \%, P\right.$-heterogeneity $\left.=0 \cdot 68\right)$. AKI events, both serious and non-serious, were reported variably across individual trials and were not adjudicated (Table S5). Overall effects of SGLT2 inhibition on major kidney outcomes are summarised in Figure 5.

Subgroup analyses

The effect on the outcome of substantial loss of kidney function, ESKD or death due to kidney disease was reported according to eGFR and UACR subgroups, and according to baseline use of

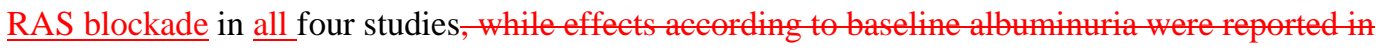
twostudies.. There was some evidence that the magnitude of relative benefit might be attenuated across progressively lower eGFR subgroups $(P$-trend=0·073; Figure 3$)$. However, there was still clear, separately significant evidence of benefit for all eGFR subgroups, including for participants with a baseline eGFR $<45 \mathrm{~mL} / \mathrm{min} / 1 \cdot 73 \mathrm{~m}^{2}$, where a $30 \%$ relative risk reduction was observed (RR $0 \cdot 70,95 \%$ CI $0 \cdot 54-0 \cdot 91, p=0 \cdot 008 \underline{0})$. There were also clear and consistent benefits in participants with eGFR above and below $60 \mathrm{~mL} / \mathrm{min} / 1.73 \mathrm{~m}^{2}(P$-heterogeneity=0.28 $\underline{33}$; Figure S2). Results for tests of heterogeneity altered slightly in the different sensitivity analyses (Table S7) but the 
evidence of clear separate benefit for all eGFR subgroups remained constant. There was no evidence of differences in treatment effect for the composite outcome across UACR subgroups $(P$ trend $=0.6631$; Figure 3 ). The effect of SGLT2 inhibitors was also consistent irrespective of the use of renin-angiotensin system blockade at baseline $(P$-heterogeneity $=0 \cdot 3177$; Figure S3).

\section{Absolute effects on eGFR slope}

The absolute effect of SGLT2 inhibitors on annualised long-term eGFR slope was reported in three studies (is summarised in Table S8. The DECLARE-TIMI 58 trial reported mean eGFR over time as a prespecified outcome instead of annualised eGFR slope. The rate of eGFR decline in placebo treated participants varied between trials from $-0.85 \mathrm{~mL} / \mathrm{min} / 1.73 \mathrm{~m}^{2} /$ year in the CANVAS Program to $-4.59 \mathrm{~mL} / \mathrm{min} / 1.73 \mathrm{~m}^{2} /$ year in the CREDENCE trial. As a result, annual placebo-subtracted differences in eGFR also differed, with the greatest absolute benefit in terms of eGFR decline observed in CREDENCE trial $\left(2 \cdot 74 \mathrm{~mL} / \mathrm{min} / 1 \cdot 73 \mathrm{~m}^{2} /\right.$ year, $95 \%$ CI $\left.2 \cdot 37-3 \cdot 11, p<0 \cdot 0001\right)$. Data by eGFR and UACR subgroups were reported in the CANVAS Program and EMPA REG OUTCOME trial, and are displayed in Table S8.

\section{DISCUSSION}

The development of kidney failure is among the most important consequences of diabetic kidney disease, and is of great concern to patients. The evidence from completed trials summarised in this review clearly shows that SGLT2 inhibitors definitively reduce the risk of kidney failuredialysis,

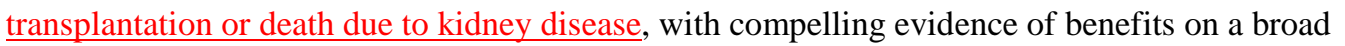
range of other clinically important kidney outcomes. Importantly, renoprotection is achieved across all levels of kidney function down to eGFR $30 \mathrm{~mL} / \mathrm{min} / 1.73 \mathrm{~m}^{2}$, with clear benefits even for the subgroup with baseline eGFR between 30 to $45 \mathrm{~mL} / \mathrm{min} / 1.73 \mathrm{~m}^{2}$ in whom these drugs are currently not approved for use in most countries. The clear protective effect against AKI allays early concerns about the risk of adverse effects consequent upon the haemodynamic mechanism of action 
of this class of agent. Furthermore, the inclusion of CREDENCE, a trial that mandated the use of RAS blockade, shows that the benefits of SGLT2 inhibitors are cumulative with those of RAS blockade. These results provide the strongest evidence yet that SGLT2 inhibition should be routinely offered to individuals with $\mathrm{T} 2 \mathrm{DM}$ at risk of progressive kidney disease.

The glycaemic efficacy of SGLT2 inhibitors is directly proportional to glomerular filtration rate,, 28 but whether the renoprotective effects are modified by declining kidney function has been less clear. A recent meta-analysis of the cardiovascular outcome trials suggested that the renoprotective effect of SGLT2 inhibitors attenuates with declining kidney function. ${ }^{14}$ However too few participants with baseline eGFR $<60 \mathrm{~mL} / \mathrm{min} / 1.73 \mathrm{~m}^{2}$ were studied to adequately assess trend by eGFR. Additionally, whether Most individual trials included few-participants with starting eGFR $<45 \mathrm{~mL} / \mathrm{min} / 1 \cdot 73 \mathrm{~m}^{2}$ derive benefits with respect to kidney outcomes, despite substantially attenuated glycaemic efficacy, has also been at baseline and were inadequately powered to test for effect modification by eGFR or albuminuria. Theunclear. The accumulated trial evidence, including the CREDENCE trial in which approximately $60 \%$ of participants had a baseline eGFR $<60$ $\mathrm{mL} / \mathrm{min} / 1 \cdot 73 \mathrm{~m}^{2}$, has made it possible to robustly explore possible modifying effects. While our findings raise the possibility that the magnitude of relative (but not absolute) benefit might attenuate somewhat across progressively lower eGFR subgroups, these results clearly demonstrate that renoprotection is achieved across the entire spectrum of eGFR levels studied to date, down to an eGFR of $30 \mathrm{~mL} / \mathrm{min} / 1 \cdot 73 \mathrm{~m}^{2}$. SGLT2 inhibitors are currently not indicated in people with eGFR $<45 \mathrm{ml} / \mathrm{min} / 1 \cdot 73 \mathrm{~m}^{2}$ in most countries, largely due to limited glycaemic efficacy. ${ }^{29}$ As these individuals are at much greater risk of kidney failure, the absolute benefits of SGLT2 inhibition are likely to be at least as large as for people with higher eGFR. ${ }^{30}$ With evidence of renoprotection now available from the cumulated trials, these restrictions are called into question, suggesting that many more individuals at high risk of major kidney outcomes are likely to benefit from treatment, and that trials in people with even more advanced kidney disease are warranted. The absence of effect 
modification by baseline albuminuria contrasts with the findings from trials of RAS blockade. $\frac{31-34}{34}$

These data suggest that mechanisms other than those associated with albuminuria reduction might also be important. Furthermore, renoprotection with SGLT2 inhibitors appears consistent irrespective of baseline use of RAS blockade. Taken together, these findings suggest that SGLT2 inhibition should provide benefit for a broader patient population.

A plausible mechanistic explanation for the renoprotective effect of SGLT2 inhibitors is correction of aberrant glomerular haemodynamics induced by hyperglycaemia, which drive progressive nephron loss. $\frac{35,36}{}$ Blocking sodium re-uptake in the proximal tubule has been suggested to restore delivery of sodium to the macula densa, leading to afferent arteriolar constriction and a reduction in

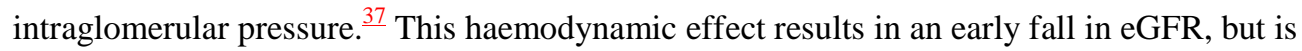
followed by marked protection against decline in kidney function, with reversal of the haemodynamic effect achieved upon drug cessation. $\frac{12,38}{}$ The effect parallels that observed with RAS blockade, the only other treatment effective in slowing the progression of diabetic kidney disease, and suggests some commonality in a mechanism of action based upon reducing intraglomerular pressure - SGLT2 inhibitors by enhancing afferent arteriolar vasoconstriction and RAS blockers by increasing efferent arteriolar vasodilatation. ${ }^{39} \underline{\text { While this may be the most }}$ plausible mechanistic explanation for the renoprotective effect of these agents, the effect on kidney haemodynamics has only been mechanistically studied in patients with type 1 diabetes with wholekidney hyperfiltration; whether these effects are also observed in patients with type 2 diabetes is not currently known. ${ }^{37}$ Other pathways by which SGLT2 inhibitors may protect the kidney an area of active study and include (but are not limited to): enhancing oxygenation of the kidney (through a reduction in tubular energy requirements), metabolic and anti-inflammatory effects, albuminuria lowering, and direct effects on glomerular endothelial function, and are an area of active study. $\frac{28,40}{}$ 
Protection against AKI is a welcome finding given early concerns about a potential increase in risk. These findings strengthen those of a previous meta-analysis of the cardiovascular outcome trials. ${ }^{16}$ While serious and non-serious AKI events were investigator reported, collected variably and not adjudicated, the large number of events and consistency of effect across the trials is striking and gives confidence to the observation that SGLT2 inhibitors provide protection against AKI. The mechanism is unknown, but could involve reduced energy expenditure in the proximal tubule, thus improving oxygenation and reducing the susceptibility of tubular cells to acute ischemic or volumerelated insults. $\frac{28,41,42}{}$ Clearly any reduction in the risk of AKI with SGLT2 inhibition must be considered in the context of other adverse effects that might also occur during an acute intercurrent illness (such as ketoacidosis), and further work is needed to better understand the mechanism(s) by which SGLT2 inhibitors reduce the risk of AKI and how this evidence might be applied in practice.

The validity of these overview results is reinforced by the high quality of the included studies, but there are a number of limitations that should be considered when interpreting our findings. We included only event-driven cardiovascular or kidney outcome trials with substantial accrued followup time. This was necessary as our main interest was in assessing kidney failure eventsthe need for dialysis, transplantation or death due to kidney disease, which wasare unlikely to be observed or responsive to therapy in trials of short duration. A substantial single trial contributed a substantial proportion of the data were derived from a single study of canagliflozin (CREDENCE trial) that was stopped early, which may increase the risk of overestimating treatment effects, ${ }^{43}$ however the consistency with the results from the other trials reduces that risk, and is an important finding of this review. While-using a single agent,_conducted in the population at highest risk of kidney failure. $t$ The consistency of effects among other members of the class therefore remains somewhat uncertain $_{2}$ although there is currently no evidence of substantive heterogeneity-The broader generalizability of these results may also be somewhat affected by the characteristics of participants in the included studies, and thus future work to assess the use of these agents in routine clinical 
practice will be important. Data for several outcomes were not available for all studies and the Ddata on AKI might be less robust than for other endpoints, due to variances in the collection and reporting of this outcome. The effects of SGLT2 inhibition on kidney (and cardiovascular and safety) outcomes in patients with eGFR $<30 \mathrm{~mL} / \mathrm{min} / 1 \cdot 73 \mathrm{~m}^{2}$ also remains an important and unanswered question, as does the comparative effectiveness of SGLT2 inhibitors against other glucose lowering agents such as glucagon-like peptide-1 (GLP-1) receptor agonists that have also demonstrated promising effects on kidney outcomes. ${ }^{44}$

Other kidney outcome trials for dapagliflozin (DAPA-CKD, NCT03036150) and empagliflozin (EMPA-KIDNEY, NCT03594110) are underway, and are expected to further enrich our understanding of the role of SGLT2 inhibitors for the prevention of kidney failure. ${ }^{24,45}$ Both trials are recruiting participants with and without T2DM on the basis of the hypothesized non-glycaemic mechanism(s) of renoprotection. Additionally the SCORED trial (NCT03315143) is testing the effects of sotagliflozin, a combined SGLT1 and SGLT2 inhibitor, on a primary cardiovascular endpoint (with other secondary kidney outcomes prespecified) in participants with T2DM and reduced kidney function. These trials will include participants with starting eGFR as low as 20 $\mathrm{mL} / \mathrm{min} / 1.73 \mathrm{~m}^{2}$ and will thus provide some important information on the effects of SGLT2 inhibition in patients with more advanced kidney disease. Future studies evaluating the combination of SGLT2 inhibitors with other glucose lowering agents that have shown beneficial impacts on kidney function, such as GLP-1 receptor agonists, are another potential area of interest, particularly in patients with established diabetic kidney disease. ${ }^{46}$

In conclusion, SGLT2 inhibition reduces the risk of dialysis, transplantation or death due to kidney disease, in people with T2DM and a broad range of kidney risk. These data provide substantive evidence supporting the use of SGLT2 inhibitors to prevent clinically important, patient-level kidney outcomes in individuals with T2DM. 


\section{Contributions}

BLN, VP, and MJJ contributed to the concept and design of this study. BLN contributed to the literature search, data extraction, risk of bias assessment, data analysis, interpretation, and writing of the manuscript. TY contributed to the literature search, data extraction, risk of bias assessment, interpretation, and drafting of the manuscript. LB contributed to the statistical analysis, interpretation, and critical review of the manuscript. HJLH, BN, VP, KWM, DMC, DCW, CA, SB, and AL contributed to the interpretation, writing, and critical review of the manuscript. BLN and MJJ drafted the first version of the manuscript and all authors contributed to revisions. All authors had full access to the data and take responsibility for the integrity of the data and accuracy of the data analysis.

\section{Acknowledgements}

The authors wish to acknowledge Associate Professor Stephen Wiviott, principle investigator of the DECLARE-TIMI 58 trial, as well as the investigators and sponsors of individual studies included in this systematic review and meta-analysis (Boehringer Ingelheim, Janssen, and AstraZeneca) who provided additional data and clarified outcome definitions when required. BLN is supported by an Australian National Health and Medical Research Council Postgraduate Scholarship, a University Postgraduate Award from UNSW Sydney, the Graduate Research Fund at Lincoln College and an Oxford Australia Clarendon Scholarship from the University of Oxford. BN has received research support from the Australian National Health and Medical Research Council Principal Research Fellowship. VP is receiving research support from the Australian National Health and Medical Research Council (Senior Research Fellowship and Program Grant). MJJ is supported by a Medical Research Future Fund Next Generation Clinical Researchers Program Career Development Fellowship.

\section{Declarations of interest}


BLN has received travel support from Janssen. TY has received sponsorship to attend meetings by

Eli Lily and Novo Nordisk. HJLH has served as a consultant for Abbvie, Astellas, AstraZeneca,

Boehringer Ingelheim, Fresenius, Janssen and Merck; and has received grant support from

AstraZeneca and Boehringer Ingelheim, with all honoraria paid to his institution. BN has received research support from Janssen, Roche, Servier, and Merck Schering Plough; and is serving on advisory boards and/or has involvement in CME pro- grams for Abbott, Janssen, Novartis, Pfizer, Roche and Servier, with any consultancy, honoraria, or travel support paid to his institution. VP is serving on Steering Committees for AbbVie, Boehringer Ingelheim, GlaxoSmithKline, Janssen and Pfizer; and is serving on advisory boards and/or speaking at scientific meetings for AbbVie, Astellas, AstraZeneca, Bayer, Baxter, Bristol-Myers Squibb, Boehringer Ingelheim, Durect, Eli Lilly, Gilead, GlaxoSmithKline, Janssen, Merck, Novartis, Novo Nordisk, Pfizer, Pharmalink, Relypsa, Roche, Sanofi, Servier and Vitae. KWM reports receiving grants from Afferent, Amgen, Apple Inc., AstraZeneca, Cardiva Medical, Daiichi, Ferring, Google (Verily), Johnson \& Johnson, Luitpold, Medtronic, Merck, Novartis, Sanofi, St Jude and Tenax, receiving personal fees from Ablynx, AstraZeneca, Baim Institute, Boehringer Ingelheim, Bristol-Myers Squibb, Cardiometabolic Health Congress, Elsevier, GlaxoSmithKline, Johnson \& Johnson, Medscape, Merck, Mitsubishi, Myokardia, Novartis, Oculeve, Portola, Radiometer, Springer Publishing, Theravance, University of California San Francisco and WebMD and having equity in BioPrint Fitness. DMC has served on clinical events committees or data safety and monitoring boards for PLC Medical, AstraZeneca, Allena Pharmaceuticals, and Merck. He has served on Steering Committees for Zoll Medical and Janssen Pharmaceuticals. He has reported consulting fees or travel from Daichi Sankyo, Fresenius and Medtronic/Coviden. DCW reports having received consultancy fees from Akebia Therapeutics, Amgen, AstraZeneca, Boehringer Ingelheim, GlaxoSmithKline, Janssen, Ono, Napp, Mundipharma and Vifor Fresenius; speaker honoraria from Amgen and Vifor Fresenius; and research support from AstraZeneca. MJJ is responsible for research projects that have received unrestricted funding from Gambro, Baxter, CSL, Amgen, Eli 
Lilly and Merck; has served on advisory boards sponsored by Akebia, Baxter and Boehringer Ingelheim; has spoken at scientific meetings sponsored by Janssen, Amgen and Roche, with any consultancy, honoraria or travel support paid to her institution. LB and SB report being full-time employees of The George Institute for Global Health, the parent organization of George Clinical, a contract research organization that provides research services to Janssen for trials of SGLT2 inhibitors. CA, and AL report no relevant declarations of interest. VP, KWM, HLJH, MJJ, BN, DCW, AL, and DMC were on the steering committee for a kidney outcome trial of an SGLT2 inhibitor (canagliflozin), with VP and KWM serving as Chair and Co-Chair. 


\section{REFERENCES}

1 Liyanage $\mathrm{T}$, Ninomiya $\mathrm{T}$, Jha $\mathrm{V}$, et al. Worldwide access to treatment for end-stage kidney disease: a systematic review. Lancet 2015; 385: 1975-82.

2 Thomas MC, Cooper ME, Zimmet P. Changing epidemiology of type 2 diabetes mellitus and associated chronic kidney disease. Nat Rev Nephrol 2016; 12: 73-81.

3 Neuen BL, Chadban SJ, Demaio AR, Johnson DW, Perkovic V. Chronic kidney disease and the global NCDs agenda. BMJ Global Health 2017; e000380.

4 Lewis EJ, Hunsicker LG, Bain RP, Rohde RD. The effect of angiotensin-converting-enzyme inhibition on diabetic nephropathy. $N$ Engl J Med 1993; 329: 1456-62.

5 Lewis EJ, Hunsicker LG, Clarke WR, et al. Renoprotective effect of the angiotensin-receptor antagonist irbesartan in patients with nephropathy due to type 2 diabetes. $N$ Engl J Med 2001; 345: $851-60$.

6 Brenner BM, Cooper ME, de Zeeuw D, et al. Effects of losartan on renal and cardiovascular outcomes in patients with type 2 diabetes and nephropathy. $N$ Engl J Med 2001; 345: 861-9.

7 Gross JL, De Azevedo MJ, Silveiro SP, Canani LH, Caramori ML, Zelmanovitz T. Diabetic nephropathy: diagnosis, prevention, and treatment. Diabetes Care 2005; 28: 164-76.

$8 \quad$ Webster AC, Nagler EV, Morton RL, Masson P. Chronic kidney disease. Lancet 2017; 389: $1238-52$.

9 Heerspink HJ, Perkins BA, Fitchett DH, Husain M, Cherney DZ. Sodium glucose cotransporter 2 inhibitors in the treatment of diabetes: cardiovascular and kidney effects, potential mechanisms and clinical applications. Circulation 2016; 134: 752-72.

10 Perkovic V, de Zeeuw D, Mahaffey KW, et al. Canagliflozin and renal outcomes in type 2 diabetes: results from the CANVAS Program randomised clinical trials. Lancet Diabetes Endocrinol 2018; 6: 691-704.

11 Wanner C, Inzucchi SE, Lachin JM, et al. Empagliflozin and progression of kidney disease in type 2 diabetes. N Engl J Med 2016; 375: 323-34.

12 Neuen BL, Ohkuma T, Neal B, et al. Cardiovascular and Renal Outcomes With Canagliflozin According to Baseline Kidney Function: Data from the CANVAS Program. Circulation 2018; 138: $1537-50$.

13 Wanner C, Lachin JM, Inzucchi SE, et al. Empagliflozin and clinical outcomes in patients with type 2 diabetes, established cardiovascular disease and chronic kidney disease. Circulation 2017; 137: 119-29.

14 Zelniker TA, Wiviott SD, Raz I, et al. SGLT2 inhibitors for primary and secondary prevention of cardiovascular and renal outcomes in type 2 diabetes: a systematic review and meta-analysis of cardiovascular outcome trials. Lancet 2018; 393: 31-9.

15 Mosenzon O, Wiviott SD, Cahn A, et al. Effects of dapagliflozin on development and progression of kidney disease in patients with type 2 diabetes: an analysis from the DECLARE-TIMI 58 randomised trial. Lancet Diabetes Endocrinol 2019; published online June. DOI:10.1016/S2213-8587(19)30180-9. 
16 Gilbert RE, Thorpe KE. Acute Kidney Injury with sodium-glucose linked cotransporter-2 inhibitors: a meta-analysis of cardiovascular outcome trials. Diabetes Obes Metab 2019. DOI:doi: 10.1111/dom.13754.

17 Perkovic V, Jardine MJ, Neal B, et al. Canagliflozin and Renal Outcomes in Type 2 Diabetes and Nephropathy. N Engl J Med 2019.

18 Higgins JP, Altman DG, Gøtzsche PC, et al. The Cochrane Collaboration's tool for assessing risk of bias in randomised trials. BMJ 2011; 343: d5928.

19 Wanner C, Inzucchi SE, Zinman B. Empagliflozin and progression of kidney disease in type 2 diabetes. N Engl J Med 2016; 375: 1801-2.

20 Neal B, Perkovic V, Mahaffey KW, et al. Optimizing the analysis strategy for the CANVAS Program: A prespecified plan for the integrated analyses of the CANVAS and CANVAS-R trials. Diabetes Obes Metab 2017; 19: 926-35.

21 Perkovic V, de Zeeuw D, Mahaffey KW, et al. Canagliflozin and renal outcomes in type 2 diabetes: results from the CANVAS Program randomised clinical trials. Lancet Diabetes Endocrinol 2018; 6: 691-704.

22 Wanner C, Heerspink HJ, Zinman B, et al. Empagliflozin and kidney function decline in patients with type 2 diabetes: a slope analysis from the EMPA-REG outcome trial. J Am Soc Nephrol 2018; 29: 2755-69.

23 Mayer GJ, Wanner C, Weir MR, et al. Analysis from the EMPA-REG OUTCOME((R)) trial indicates empagliflozin may assist in preventing the progression of chronic kidney disease in patients with type 2 diabetes irrespective of medications that alter intrarenal hemodynamics. Kidney Int 2019; published online March. DOI:10.1016/j.kint.2019.02.033.

24 Herrington WG, Preiss D, Haynes R, et al. The potential for improving cardio-renal outcomes by sodium-glucose co-transporter-2 inhibition in people with chronic kidney disease: a rationale for the EMPA-KIDNEY study. Clin Kidney J 2018; 11: 749-61.

25 Neal B, Perkovic V, Mahaffey KW, et al. Canagliflozin and cardiovascular and renal events in type 2 diabetes. $N$ Engl J Med 2017; 377: 644-57.

26 Zinman B, Wanner C, Lachin JM, et al. Empagliflozin, cardiovascular outcomes, and mortality in type 2 diabetes. N Engl J Med 2015; 373: 2117-28.

27 Wiviott SD, Raz I, Bonaca MP, et al. Dapagliflozin and cardiovascular outcomes in type 2 diabetes. N Engl J Med 2018; 380: 347-57.

28 Heerspink HJ, Kosiborod M, Inzucchi SE, Cherney DZ. Renoprotective effects of sodiumglucose cotransporter-2 inhibitors. Kidney Int 2018; 94: 26-39.

29 Toyama T, Neuen BL, Jun M, et al. Effect of SGLT2 inhibitors on cardiovascular, renal and safety outcomes in patients with type 2 diabetes mellitus and chronic kidney disease: a systematic review and meta-analysis. Diabetes Obes Metab 2019; 21: 1237-50.

30 Gansevoort RT, Matsushita K, Van Der Velde M, et al. Lower estimated GFR and higher albuminuria are associated with adverse kidney outcomes. A collaborative meta-analysis of general and high-risk population cohorts. Kidney Int 2011; 80: 93-104. 
31 Jafar TH, Schmid CH, Landa M, et al. Angiotensin-converting enzyme inhibitors and progression of nondiabetic renal disease: a meta-analysis of patient-level data. Ann Intern Med 2001; 135: 73-87.

32 Kent DM, Jafar TH, Hayward RA, et al. Progression risk, urinary protein excretion, and treatment effects of angiotensin-converting enzyme inhibitors in nondiabetic kidney disease. $J$ Am Soc Nephrol 2007; 18: 1959-65.

33 Molitch ME, Adler AI, Flyvbjerg A, et al. Diabetic kidney disease: a clinical update from Kidney Disease: Improving Global Outcomes. Kidney Int 2015; 87: 20-30.

34 Cherney DZ, Zinman B, Inzucchi SE, et al. Effects of empagliflozin on the urinary albuminto-creatinine ratio in patients with type 2 diabetes and established cardiovascular disease: an exploratory analysis from the EMPA-REG OUTCOME randomised, placebo-controlled trial. Lancet Diabetes Endocrinol 2017; 5: 610-21.

35 Alicic RZ, Rooney MT, Tuttle KR. Diabetic Kidney Disease Challenges, Progress, and Possibilities. Clin J Am Soc Nephrol 2017; 12: 2032-45.

36 Tonneijck L, Muskiet MH, Smits MM, et al. Glomerular hyperfiltration in diabetes: mechanisms, clinical significance, and treatment. J Am Soc Nephrol 2017; 28: 1023-39.

37 Cherney DZ, Perkins BA, Soleymanlou N, et al. Renal hemodynamic effect of sodium-glucose cotransporter 2 inhibition in patients with type 1 diabetes mellitus. Circulation 2014; 129: $\underline{587-97 .}$

38 Fioretto P, Del Prato S, Buse JB, et al. Efficacy and Safety of Dapagliflozin in Patients with Type 2 Diabetes and Moderate Renal Impairment (Chronic Kidney Disease Stage 3A): The DERIVE Study. Diabetes Obes Metab 2018; 20: 2532-40.

39 Tuttle KR. Back to the future: Glomerular hyperfiltration and the diabetic kidney. Diabetes 2017; 66: 14-6.

40 Heerspink HJ. Sodium glucose co-transporter 2 inhibition: a new avenue to protect the kidney. Nephrol Dial Transplant 2019; DOI: 10.1093/ndt/gfz033

41 Chang Y-K, Choi H, Jeong JY, et al. Dapagliflozin, SGLT2 inhibitor, attenuates renal ischemia-reperfusion injury. PLoS One 2016; 11: e0158810.

42 O'Neill J, Fasching A, Pihl L, Patinha D, Franzén S, Palm F. Acute SGLT inhibition normalizes $\mathrm{O} 2$ tension in the renal cortex but causes hypoxia in the renal medulla in anaesthetized control and diabetic rats. Am J Physiol Renal Physiol 2015; 309: F227-34.

43 Viele K, McGlothlin A, Broglio K. Interpretation of clinical trials that stopped early. JAMA 2016; 15: 1646-7.

44 Zelniker TA, Wiviott SD, Raz I, et al. Comparison of the Effects of Glucagon-Like Peptide Receptor Agonists and Sodium-Glucose Co-Transporter 2 Inhibitors for Prevention of Major Adverse Cardiovascular and Renal Outcomes in Type 2 Diabetes Mellitus: A Systematic Review and Meta-Analysis of Cardiovascular Outcomes Trials. Circulation 2019; 139: 2022 $\underline{31 .}$

45 Pecoits-Filho R, Perkovic V. Are SGLT2 Inhibitors Ready for Prime Time for CKD? Clin J 
Am Soc Nephrol 2017; 13: 318-20.

46 Goncalves E, Bell DS. Combination treatment of SGLT2 inhibitors and GLP-1 receptor agonists: symbiotic effects on metabolism and cardiorenal risk. Diabetes Ther 2018; 9: 919 $\underline{26}$. 


\section{FIGURE LEGENDS}

Figure 1. Effect of SGLT2 inhibitors on kidney failure (dialysis, transplantation-or death-due to kidney disease)-dialysis, transplantation or death due to kidney disease

SGLT2: sodium-glucose cotransporter 2 inhibitor; RR: relative risk; CI: confidence interval; N/A: not available.

Figure 2. Effect of SGLT2 inhibitors on (A) ESKD, (B) substantial loss of kidney function, ESKD, or death due to kidney disease and (C) substantial loss of kidney function, ESKD, or death due to cardiovascular or kidney disease-

ESKD: end-stage kidney disease; SGLT2: sodium-glucose cotransporter 2 inhibitor; RR: relative risk; CI: confidence interval; N/A: not available.

Figure 3. Effect of SGLT2 inhibitors on substantial loss of kidney function, ESKD or death due to kidney disease, stratified by baseline (A)-baseline GGFR $_{2}$ and (B) UACR-subgroups, and $(C)$ use of renin-angiotensin system blockade

ESKD: end-stage kidney disease; eGFR: estimated glomerular filtration rate; UACR: urinary albumin:creatinine ratio; SGLT2: sodium-glucose cotransporter 2 inhibitor; RR: relative risk; CI:

confidence interval; N/A: not available.

Figure 4. Effect of SGLT2 inhibitors on acute kidney injury-

SGLT2: sodium-glucose cotransporter 2 inhibitor; RR: relative risk; CI: confidence interval.

Figure 5. Summary of the effects of SGLT2 inhibition on major kidney outcomes SGLT2: sodium-glucose cotransporter 2 inhibitor; RR: relative risk; CI: confidence interval 


\section{$\checkmark$ The George Institute} for Global Health
Australia
Level 5, 1 King Street Newtown NSW 2042 AUSTRALIA

PO Box M201 Missenden Road NSW 2050 AUSTRALIA

T: +61280524300

F: +61280524301

info@georgeinstitute.org.au www.georgeinstitute.org

\section{June 2019}

Dr Marta Koch PhD

Editor-in-Chief

Lancet Diabetes and Endocrinology

Dear Marta,

On behalf of my coauthors, thank you for reviewing our manuscript "SGLT2 inhibitors for the prevention of kidney failure in patients with type 2 diabetes: a systematic review and meta-analysis" for potential publication in Lancet Diabetes and Endocrinology. We are grateful for the time and effort that you and the reviewers have taken to consider our paper.

We have revised the manuscript and addressed the issues raised. Enclosed in this letter is a description of our responses to your comments. We hope that this revision meets your requirements. I confirm all co-authors agreed to these changes and have approved resubmission. Thank you again for consideration our manuscript and I look forward to hearing from you.

Sincerely,

Meg Jardine

Program Head, Innovative Kidney Research

Asssociate Professor of Medicine, UNW Sydney

Staff Specialist Nephrology, Concord Repatriation General Hospital

Head, Renal Trial, George Clinical 


\section{EDITORS' SPECIFIC POINTS:}

*As noted by Reviewer \#3 and others, there are several previous meta-analyses that have been done on the topic covered here - specifically there is the meta of the CVOTs from the TIMI group published in The Lancet last November (https://www.thelancet.com/journals/lancet/article/PIIS0140-6736(18)32590-

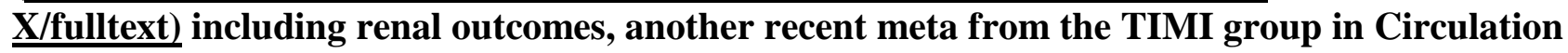
comparing SGLT inhibitors and GLP-1 receptor agonists

(https://www.ahajournals.org/doi/full/10.1161/CIRCULATIONAHA.118.038868) also including renal outcomes, and recent meta on AKI in the CVOTs of SGLT2 inhibitors (https://onlinelibrary.wiley.com/doi/full/10.1111/dom.13754). These papers ought to be cited and discussed in the revised paper, including emphasising what the present paper adds beyond those previous metas (while the main advance is the inclusion of data from CREDENCE, any other novel aspects from the present work should also be emphasised).

As suggested, we have cited these works and incorporated them into the research in context, introduction and discussion sections. More specifically, we have used these papers to highlight what additional contribution this meta-analysis makes to the literature, which most notably includes the addition of the first dedicated kidney outcome trial (CREDENCE), but also a range of other important issues as described below.

Research in context, evidence before this study:

"SGLT2 inhibitors are also not currently approved for use in patients with estimated glomerular filtration rate (eGFR) less than 45 or $60 \mathrm{~mL} / \mathrm{min} / 1.73 \mathrm{~m}^{2}$ in most countries, primarily because their glucose lowering effect is substantially dependent on kidney function. A recent meta-analysis of these trials has reported that the renoprotective effect of SGLT2 attenuates with declining kidney function. However, less than a sixth of participants studied had a baseline eGFR less than 60 $\mathrm{mL} / \mathrm{min} / 1 \cdot 73 \mathrm{~m}^{2}$, and even fewer had baseline eGFR less than $45 \mathrm{~mL} / \mathrm{min} / 1 \cdot 73 \mathrm{~m}^{2}$. The ability to robustly assess effects in people with reduced kidney function was therefore limited, especially since few patient-level kidney outcomes occurred. While collectively these trials have suggested that SGLT2 inhibitors might protect against acute kidney injury, the safety of these agents in patients at high risk of adverse kidney outcomes has remained a concern."

Introduction:

"A recent meta-analysis of cardiovascular outcome trials suggested that the effect of SGLT2 inhibitors on kidney outcomes attenuates with declining eGFR. However, less than a sixth of participants in this analysis had baseline eGFR $<60 \mathrm{~mL} / \mathrm{min} / 1.73 \mathrm{~m} 2$, and thus the ability to robustly assess effect modification by kidney function has been limited. Additionally, because very few participants with starting eGFR $<45 \mathrm{~mL} / \mathrm{min} / 1.73 \mathrm{~m} 2$ have been studied, it has also been unclear whether these patients derive protection against kidney outcomes, since the glycaemic efficacy of these agents is substantially attenuated in this population. Similarly, few participants with higher levels of albuminuria have been studied, and therefore the consistency of treatment effect across different levels of albuminuria is unclear. While these trials have collectively suggested protection against acute kidney injury, the safety of these agents in patients at higher risk of adverse kidney outcomes has not been well established."

Discussion:

"A recent meta-analysis of the cardiovascular outcome trials suggested that the renoprotective effect of SGLT2 inhibitors attenuates with declining kidney function. However too few participants with baseline eGFR $<60 \mathrm{~mL} / \mathrm{min} / 1.73 \mathrm{~m}^{2}$ were studied to adequately assess trend by eGFR. Additionally, whether participants with starting eGFR $<45 \mathrm{~mL} / \mathrm{min} / 1 \cdot 73 \mathrm{~m}^{2}$ derive benefits 
with respect to kidney outcomes, despite substantially attenuated glycaemic efficacy, has also been unclear."

"The effects of SGLT2 inhibition on kidney (and cardiovascular and safety) outcomes in patients with eGFR $<30 \mathrm{~mL} / \mathrm{min} / 1.73 \mathrm{~m}^{2}$ also remains an important and unanswered question, as does the comparative effectiveness of SGLT2 inhibitors against other glucose lowering agents such as glucagon-like peptide-1 (GLP-1) receptor agonists that have also demonstrated promising effects on kidney outcomes."

*As Reviewer \#3 notes, some data seem to be missing from some of the trials. In order to prioritise the present paper, we would need it to be as complete as possible. A renal analysis from DECLARE-TIMI 58 was published yesterday

(https://www.thelancet.com/journals/landia/article/PIIS2213-8587(19)30180-9/fulltext), which might help a little, although perhaps not completely as this did not include detailed UACR data. Have you approached the authors to request the data needed to make these analyses complete? If not, might it be possible to wait until these data are available to ensure these analyses are complete? Similarly for the event rate data from EMPA-REG OUTCOME, have you approached the authors to request these data?

We contacted study investigators and trial sponsors wherever possible to obtain additional data or clarify outcomes.

During the review process, we contacted the DECLARE-TIMI 58 investigators, who provided data for the outcome of dialysis, transplantation or death due to kidney disease. As a result, there is now complete data across all four studies for the primary outcome in this review.

The cardio-renal composite outcome of substantial loss of kidney function, ESKD, or death due to cardiovascular or kidney disease in the EMPA-REG OUTCOME trial has not been previously published. The effect on this outcome in the EMPA-REG OUTCOME trial was obtained through correspondence with the trial sponsor (Figure 2, panel C). This allows for the complete reporting of this outcome across all the included studies.

The renal composite outcome of substantial loss of kidney function, ESKD, or death due to kidney disease from the CANVAS Program by baseline UACR subgroups has also not been previously published. These data were analysed specifically for this manuscript through the provision of data from the CANVAS Program authors.

Additionally, the exact definitions of acute kidney injury used each of the trials (Table S5), and adjudication procedures were also clarified with study sponsors.

As suggested, we have incorporated the data from the recent publication of the DECLARE-TIMI 58 renal outcomes paper into our manuscript. This includes detailed data for subgroups, including by baseline eGFR, UACR, and use of renin-angiotensin system blockade.

As a result, there is now complete data across the four studies for every outcome in the manuscript. There is also complete outcome data for all the subgroup analysis by baseline eGFR, UACR and use of renin-angiotensin system blockade. We have amended the manuscript to clarify how data were obtained and acknowledged investigators and trial sponsors.

\section{Methods:}

"Study sponsor and investigators were contacted to obtain additional trial-level data and clarify outcome definitions when required." 


\section{Acknowledgements:}

"The authors wish to acknowledge Associate Professor Stephen Wiviott, principle investigator of the DECLARE-TIMI 58 trial, as well as the investigators and sponsors of individual studies included in this systematic review and meta-analysis (Boehringer Ingelheim, Janssen, and AstraZeneca) who provided additional data and clarified outcome definitions when required."

Unfortunately, event rates per 1000 patient years are variably reported across the primary trial reports and secondary analyses and we were not able to obtain event rates for all studies. We are currently unaware of any reporting of event rates from the EMPA-REG OUTCOME trial for eGFR and UACR subgroups.

In contrast in the CANVAS Program publications, event rates have been reported but the number of events in each trial arm has not been for statistical reasons, which are outlined below in our response to the reviewer.

To attempt to address this, we have reported the total number of events and participants in each trial for every outcome so that the reported data is complete as possible.

*As noted by the statistical reviewer, it seems inappropriate to refer the PROSPERO registration as 'prospective' when it was done just days before submission and when the analyses were well under way. Perhaps this could be rephrased to avoid the word 'prospective' - eg, "was registered on PROSPERO before the analyses were completed".

We appreciate that these analyses were well underway by the registration date of this review, as listed on the PROSPERO website. This was due to a substantial delay between submission of a protocol to PROSPERO and it being approved and publicly listed.

In the case of this systematic review and meta-analysis, the initial protocol submission occurred on the $10^{\text {th }}$ of April 2019 (prior to the publication of the CREDENCE trial), but the protocol was not processed by PROSPERO until the $22^{\text {nd }}$ of May - more than six weeks later. PROSPERO does not acknowledge the date of protocol submission $\left(10^{\text {th }}\right.$ April $)$, only the data that it is approved $\left(22^{\text {nd }}\right.$ May).

Nevertheless, we have amended the manuscript as suggested to better reflect the conduct of the analyses, which occurred whilst the protocol was being processed, as permitted by PROSPERO.

Research in context, evidence before this study:

"The protocol for this systematic review and meta-analysis was registered in the International Prospective Register of Systematic Reviews before the analyses were completed (PROSPERO registration number CRD42019131774)."

Methods:

"The protocol for this review was submitted before the analyses were begun (10 April 2019) and was registered by the International Prospective Register of Systematic Reviews before the analyses were completed (PROSPERO registration number CRD42019131774) and can be accessed at: http://www.crd.york.ac.uk/PROSPERO/display_record.php?ID=CRD42019131774."

\section{REVIEWERS' COMMENTS:}

Reviewer \#1: This well-written and very timely manuscript by Brendon Neuen and colleagues summarizes renal outcome data from three landmark cardiovascular safety trials and one 
dedicated renal outcome trial of SGLT2i's (collectively including 38,723 randomized participants) in patients with type 2 diabetes and high cardio-renal risk. This systematic review provides the strongest evidence to date that SGLT2 inhibitors reduce the risk of endstage kidney disease, renal death, acute kidney injury and a range of other kidney outcomes in T2DM patients; albeit CREDENCE contributed to a substantial proportion of the data. Importantly, this meta-analysis provides substantive evidence that SGLT2i's induce renoprotection across the spectrum of kidney function levels, including separate evidence of benefit for T2DM patients with a baseline eGFR between 30 and $45 \mathrm{~mL} / \mathrm{min} / 1.73 \mathrm{~m} 2$. This highly relevant outcome and clinical message may resonate as to date, these drugs are not permitted for use in patients with moderate to severe kidney failure in most countries. Finally, collective data allays the concerns about the risk of AKI; rather, the analysis shows a clear protective effect (keeping in mind the variably collected and not adjudicated data) which sparks the search for mechanistic understanding and potential beyond diabetic kidney disease.

Although the results are of high interest and are generally well-presented, some critical comments and suggestions may help the authors/investigators to improve the manuscript.

\section{Minor comments}

1. General: The primary composite outcome of this systematic review and meta-analysis is "kidney failure", defined as chronic dialysis, kidney transplantation or death due to kidney disease. The title of this composite seems poorly chosen given that the latter outcome (renal death) denotes more than kidney failure alone. Please consider adjusting the wording of the primary composite. In general, all outcomes (primary and secondary) seem to be very similar in terms of composition and naming, which is at times quite confusing. For instance, the main result sentence suggests overlap that does not count up: "there were 218 occurrences of kidney failure, 310 ESKD events ... 943 episodes of AKI".

We have amended the primary outcome from "kidney failure" to "dialysis, transplantation or death due to kidney disease". We believe this clarifies the confusion about the different outcomes with similar terminology and/or components.

Abstract, methods:

"The primary outcome was dialysis, transplantation, or death due to kidney disease."

Abstract, findings:

"Data were obtained from four studies including 38,723 participants of whom 252 required dialysis or transplantation or died due to kidney disease, 335 developed end-stage kidney disease (ESKD), and 943 experienced acute kidney injury (AKI). SGLT2 inhibitors reduced the risk of dialysis, transplantation or death due to kidney disease (RR 0.67, 95\% CI 0.52-0.86, p=0.0019)"

Methods, data synthesis and analysis:

"The primary outcome of interest was chronic dialysis, kidney transplantation, or death due to kidney disease."

Results:

"Overall, there were 252 participants who required dialysis or transplantation or died due to kidney disease."

Results, primary outcome: 
"SGLT2 inhibitors reduced the risk of dialysis, transplantation or death due to kidney disease by $33 \%$ (RR 0.67, 95\% CI 0.52-0.86, $p=0 \cdot 0019$ ). The effect of SGLT2 inhibitors on this outcome was consistent across studies $\left(I^{2}=0 \%, P\right.$-heterogeneity=0.53; Figure 1$)$. “

\section{Discussion:}

"The evidence from completed trials summarised in this review clearly shows that SGLT2 inhibitors definitively reduce the risk of dialysis, transplantation or death due to kidney disease, with compelling evidence of benefits on a broad range of other clinically important kidney outcomes."

\section{Methods:}

2. General: As CREDENCE contributed to a substantial proportion of the data, and resulting conclusion and clinical message, I believe the authors should discuss this trial in slightly more detail, including its potential limitations (e.g. relatively high drug-discontinuation rate and impact of early cessation of the trial).

The drug discontinuation rate in CREDENCE was $27.3 \%$ over a median 2.6 years and was comparable to that seen with other SGLT2 inhibitor trials and other major renal outcome trials (further details listed below). Discontinuation rates would be expected to bias the results towards the null. The proportion of participants who discontinued randomized treatment along with median/mean follow-up in other SGLT2 inhibitor trials and major renal outcome trials are listed below:

\section{SGLT2 inhibitor trials}

- CREDENCE: median follow-up 2.6 years, stopped early

○ $29.9 \%$ placebo, $24.7 \%$ canagliflozin

- CANVAS Program: median follow-up 2.4 years

○ $29.9 \%$ placebo, $29.2 \%$ canagliflozin

- DECLARE-TIMI 58: median follow up 4.2 years

○ $25.1 \%$ placebo, $21.1 \%$ dapagliflozin

- EMPA-REG OUTCOME: median follow up 3.1 years

○ $29.3 \%$ placebo, $23.4 \%$ empagliflozin

\section{Renal outcome trials}

- SONAR: median follow up 2.2 years, stopped early

○ $19.0 \%$ placebo, $19.6 \%$ atrasentan

- ALTITUDE: median follow up 2.7 years, stopped early

- $29.1 \%$ placebo, $34.3 \%$ aliskiren

- NEPHRON-D: median follow-up 2.2 years, stopped early

○ $16.1 \%$ monotherapy, $20.7 \%$ combination therapy

- IDNT: mean follow-up 2.6 years

○ $23.7 \%$ of participants

- RENAAL: mean follow-up 3.4 years, stopped early

○ $53.5 \%$ placebo, $46.5 \%$ losartan

We believe that the rates of discontinuation across these trials reflect the high-risk nature of the population that these trials have been conducted in, an issue which is not limited solely to the CREDENCE trial.

We have edited the limitations section of the discussion to acknowledge that the CREDENCE trial was stopped early and the impact this may have on our findings. 
Discussion:

"A substantial proportion of the data were derived from a single study of canagliflozin (CREDENCE trial) that was stopped early, which may increase the risk of overestimating treatment effects, however the consistency with the results from the other trials reduces that risk, and is an important finding of this review. While the consistency of effects among other members of the class therefore remains somewhat uncertain, there is currently no evidence of substantive heterogeneity"

3. Abstract (Page 4, Background): The use of the word "impact" is too imprecise; perhaps use "magnitude of effect". Furthermore, I would argue that the hitherto undefined heterogeneity of effect based on baseline kidney impairment should also be mentioned here.

As suggested, we have amended the statement. We have also discussed heterogeneity of effect based on kidney function (and albuminuria) in greater detail in the introduction and discussion sections. Additionally, we have also incorporated this point into the research in context section.

Summary, background:

"The effect of sodium-glucose cotransporter 2 (SGLT2) inhibitors on kidney failure, particularly the need for dialysis and transplantation, or death due to kidney disease, has been uncertain. Previous studies have also been underpowered to robustly assess heterogeneity of effects on kidney outcomes by different levels of estimated glomerular filtration rate (eGFR) and albuminuria"

Research in context, evidence before this study:

"A recent meta-analysis of these trials has reported that the renoprotective effect of SGLT2 attenuates with declining kidney function. However, less than a sixth of participants studied had a baseline eGFR less than $60 \mathrm{~mL} / \mathrm{min} / 1.73 \mathrm{~m}^{2}$, and even fewer had baseline eGFR $<45$ $\mathrm{mL} / \mathrm{min} / 1.73 \mathrm{~m}^{2}$. The ability to robustly assess effects in people with reduced kidney function was therefore limited, especially since few patient-level kidney outcomes occurred."

4. Abstract/Methods (Pages 4 and 7): The authors state that MEDLINE and EMBASE were searched from inception to 1 March 2019; however, the CREDENCE trial was presented to the public and published in NEJM on 14 April 2019. Please adjust the search criteria.

As suggested, we have updated the search criteria. We have also included renal outcome data from the DECLARE-TIMI 58 trial, which was also recently published, and have updated all our analyses accordingly.

Research in context, evidence before this study:

"MEDLINE and EMBASE were searched from inception until June 142019 to identify potentially relevant studies."

Methods:

"We searched MEDLINE and EMBASE from inception to 14 June 2019 to identify potentially eligible studies."

5. Introduction (Page 6): Please be more exact when summarizing the potential mechanisms underlying the cardiovascular event benefits of SGLT2i's; it is unlikely that the currently mentioned pleiotropic actions solely account for these actions.

Because cardiovascular outcomes are not the primary focus of this manuscript and due to constraints in the word limit, we have omitted this statement altogether so that we could address the following comment below in greater detail. 
6. Introduction (Page 6): I would suggest to add some brief background on the main purpose of CVOT's, and in general, be keen on reporting that CV-benefits (as well as potential renal benefits) were by design shown in T2DM patients at high CV-risk or established atherosclerotic disease. Perhaps cite the following: Lancet 2019;393(10166):31-39).

We have amended the introduction and cited the Lancet manuscript as suggested. As cardiovascular outcomes are well summarized and discussed elsewhere (such as in the Lancet meta-analysis), we have chosen to focus on kidney outcomes in greater detail.

Introduction:

"Large scale randomized trials of SGLT2 inhibitors, which were originally designed to meet regulatory requirements and ensure cardiovascular safety, have demonstrated promising effects on a range of albuminuria and serum creatinine based kidney outcomes in patients with, or at high risk of, atherosclerotic cardiovascular disease."

7. Methods (Page 7): I value the use PROSPERO by the authors to help avoid duplication and reduce opportunity for reporting bias. I do note that the initial registration date (22 May 2019) was only just before (or may even post-date) the manuscript submission-date to The Lancet D\&E; please explain why registration was performed in this late stage of the reviewprocess.

As explained to our response to the editors, the initial PROSPERO protocol submission occurred on the $10^{\text {th }}$ of April 2019 (prior to the publication of the CREDENCE trial), but the protocol was not processed by PROSPERO until the $22^{\text {nd }}$ of May - more than six weeks later. PROSPERO does not acknowledge the date of protocol submission $\left(10^{\text {th }}\right.$ April $)$, only the data that it is approved $\left(22^{\text {nd }}\right.$ May). We appreciate that it is clear that these analyses were clearly well underway by the $22^{\text {nd }}$ of May, as listed on the PROSPERO website.

We have therefore edited the manuscript to more appropriately address the timing of the analyses.

Research in conxtext, evidence before this study:

"The protocol for this systematic review and meta-analysis was registered in the International Prospective Register of Systematic Reviews before the analyses were completed (PROSPERO registration number CRD42019131774)."

Methods:

"The protocol for this review was submitted before the analysis were begun (10 April 2019) and were registered in the International Prospective Register of Systematic Reviews before the analyses were completed (PROSPERO registration number CRD42019131774) and can be accessed at: http://www.crd.york.ac.uk/PROSPERO/display_record.php?ID=CRD42019131774."

8. Methods (Page 8): When detailing the use of sustained versus unsustained kidney outcomes, please specify whether the corrected renal outcome data from EMPA-REG OUTCOME were used (as presented in a separate Correspondence; i.e. Wanner C, N Engl J Med. 2016 Nov $3 ; 375(18): 1801-2)$, or data from the main renal publication were taken (Wanner $\mathbf{C}$, $\mathbf{N}$ Engl J Med. 2016 Jul 28;375(4):323-34)

We have clarified this in the methods section, as requested.

Methods: 
"We preferentially used data on sustained kidney outcomes confirmed with repeat assessment where these were reported to exclude acute changes in kidney function and initiation of dialysis for AKI, but accepted unsustained outcomes where these were the only ones reported. This was the case for the EMPA-REG OUTCOME trial, where sustained kidney outcome data were reported in separate correspondence ${ }^{19}$ following the main trial publication. ${ }^{11}$ ",

9. Results (Page 11): When describing the four separate studies, I suggest adding references, as well as the secondary analyses of these trials that were used for this meta-analysis.

We have now stated this and referenced the secondary analyses used in this meta-analysis.

Results:

"Data from a number of secondary analyses of these studies were used in this meta-analysis. ${ }^{11-}$ 13,15,19,21-24,"

10. Discussion (Pages 15-16): Although I agree with the authors that the "renal hemodynamics theory" is the most plausible mechanistic explanation for the renoprotective effect of SGLT2i's, the authors should acknowledge that the renal hemodynamic effects of these drugs are only mechanistically studied in type 1 diabetes patients with whole-kidney hyperfiltration. It should be stated that the effect of SGLT2i's on renal vascular resistance in T2DM patients are currently unknown. Of note, when discussing other pathways of kidney protection, please add the potential of SGLT2i's of reducing renal ischemia (e.g. via reduced energy requirements and increased hematocrit), as also indicated in the next section on AKI.

We have amended the manuscript as suggested.

Discussion:

"While this may be the most plausible mechanistic explanation for the renoprotective effect of these agents, the effect on kidney haemodynamics has only been mechanistically studied in patients with type 1 diabetes with whole-kidney hyperfiltration; whether these effects are also observed in patients with type 2 diabetes is not currently known.

"Other pathways by which SGLT2 inhibitors may protect the kidney an area of active study and include (but are not limited to): enhancing oxygenation of the kidney (through a reduction in tubular energy requirements), metabolic and anti-inflammatory effects, albuminuria lowering, and direct effects on glomerular endothelial function."

11. Discussion (Page 17): Please add that sotagliflozin is an SGLT1 and SGLT2 inhibitor.

Thank you for pointing this out; we have corrected the manuscript.

Discussion:

"Additionally the SCORED trial (NCT03315143) is testing the effects of sotagliflozin, a combined SGLT1 and SGLT2 inhibitor, on a primary cardiovascular endpoint (with other secondary kidney outcomes prespecified) in participants with T2DM and reduced kidney function."

12. Table 1: Please add an asterisk next to the follow-up duration of CREDENCE, to indicate that this trial was stopped early after a planned interim analysis on the recommendation of the DSMB.

We have amended Table 1 as suggested. 
Reviewer \#2 [statistical reviewer]: Thank you for the opportunity to review this submission, describing a systematic review \& meta-analysis of the SGLT2 inhibitors for the prevention of kidney failure in patients with T2DM. The review was carried out in accordance with many best practices for evidence synthesis and I am pleased to see a review that focuses on the outcomes that matter most to patients.

The SGLT2 inhibitors are one of many treatment options for patients with T2DM. This review compares the SGLT2 inhibitors (lumped together as a group) to placebo. There is no comparison to other existing (ie active) treatments. So, this review presents a small piece of the puzzle - leaving clinicians, patients and policy makers with residual uncertainty as to the place of SGLT2 inhibitors in the treatment pathway. What would have made this review useful would have been to present the SGLT2 inhibitors in the context of existing treatments (a la network meta-analysis). This would provide decision makers (clinicians, patients \& policy makers) with a powerful resource comparing both the relative comparative benefits and relative comparative harm - so that the balance between benefits and harms could be weighed up. Moreover, the paper relies solely on "relative" comparisons (SGLT2 vs placebo); it would be informative to also see absolute risk data. The "lumping" together of 4 trials - each of which involved a different SGLT2 inhibitor - also doesn't provide meaningful input for clinicians, patients or policy makers. [EDITORS' NOTE: we are happy for the authors to simply rebut this Comment from the statistical reviewer]

The authors recognise the importance of comparative effectiveness research, particularly in the context of glucose lowering agents. We agree that a network meta-analysis would indeed be useful to clinicians, patients and policy makers. While a network meta-analysis is beyond the scope of the current work, this is certainly something that we would be interested in pursuing in the future. Nevertheless, we believe this meta-analysis does address important unanswered questions, focusing on the kidney outcomes that are most important to patients. Several previous meta-analysis of SGLT2 inhibitors have also quantitatively synthesized different agents within the class, with tests for heterogeneity used to investigate the presence or absence of a class effect. The remarkably consistent evidence across the agents studied to date strongly suggests that the effect on kidney outcomes is indeed a class effect, which is being increasingly recognised in major clinical practice guidelines.

There have been no new agents approved for the treatment of diabetic kidney disease in almost two decades. Up until now, no glucose-lowering agent has been shown to reduce the risk of end-stage kidney disease due to type 2 diabetes. While SGLT2 inhibitors have demonstrated promising effects on albuminuria and creatinine based outcomes, their effect on the kidney outcomes that matter most to patients - namely, dialysis, transplantation or death due to kidney disease - has been uncertain. Additionally these results demonstrate that patients with reduced kidney function (in whom these treatments are mostly not permitted for use) stand to gain substantial kidney benefits if treated. For these reasons, we believe this meta-analysis has the potential to impact policy and practice.

A quick check of PubMed suggests this review is not the first on this topic; another MA was published in early May. Diabetes Obes Metab. 2019 May 3. doi: 10.1111/dom.13754. [Epub ahead of print]. Acute kidney injury with sodium-glucose co-transporter-2 inhibitors: A meta-analysis of cardiovascular outcome trials.

As outlined in our response to the editors, we have cited this paper along with other work on the topic to highlight novel aspects of this meta-analysis, beyond the inclusion of the CREDENCE trial.

Research in context, evidence before this study: 
"A recent meta-analysis of these trials has reported that the renoprotective effect of SGLT2 attenuates with declining kidney function. However, less than a sixth of participants studied had a baseline eGFR less than $60 \mathrm{~mL} / \mathrm{min} / 1.73 \mathrm{~m}^{2}$, and even fewer had baseline eGFR less than 45 $\mathrm{mL} / \mathrm{min} / 1.73 \mathrm{~m}^{2}$. The ability to robustly assess effects in people with reduced kidney function was therefore limited, especially since few patient-level kidney outcomes occurred. While collectively these trials have suggested that SGLT2 inhibitors might protect against acute kidney injury, the safety of these agents in patients at high risk of adverse kidney outcomes has remained a concern."

Introduction:

"While these trials have collectively suggested protection against acute kidney injury, the safety of these agents in patients at higher risk of adverse kidney outcomes has not been well established."

\section{Discussion:}

"Protection against AKI is a welcome finding given early concerns about a potential increase in risk. These findings strengthen those of a previous meta-analysis of the cardiovascular outcome trials."

Other issues that should be considered/addressed:

-All SGLT2 inhibitors were lumped together; no examination of one treatment vs another.

Through our systematic literature search, no direct head-to-head trials of different SGLT2 inhibitors reporting effects on major kidney outcomes were identified. Additionally we did not identify any head-to-head cardiovascular or kidney outcome trials of SGLT2 inhibitors versus any other glucose lowering agents that reported effects on major kidney outcomes. Our analysis of SGLT2 inhibitors by individual trial and overall class is consistent with a number of other meta-analyses of these drugs.

-Date of PROSPERO entry is May 22, 2019. This is the initial registration date, not the update date (as there has not been an update to the PROSPERO entry). This is problematic as PROSPERO is to be prospective registration....yet if this review was submitted to the Lancet in late May (and the literature search went to March 1, 2019), it's clear that the review was done prior to PROSPERO registration which is then, disingenuous. The present PROSPERO entry (May 22, 2019) also fails to indicate the current status of the review; all steps are noted as started but not completed....but if this paper has been submitted to the Lancet, then it's obvious all steps of the review are completed. Again, this flies in the face of the spirit of PROSPERO as others may initiate work on their own review, thinking they may be able to complete it before this group completes their review.

We appreciate that the entry date as listed on PROSPERO was clearly very close to the analyses being completed. This was due to a substantial delay between submission of a protocol to PROSPERO and it being approved and publicly listed.

The initial protocol submission occurred on the $10^{\text {th }}$ of April 2019 (prior to the public reporting of the CREDENCE trial), but the protocol was not processed by PROSPERO until the $22^{\text {nd }}$ of May more than six weeks later. PROSPERO does not acknowledge the date of protocol submission $\left(10^{\text {th }}\right.$ April), only the data that it is approved ( $22^{\text {nd }}$ May).

Nevertheless, we have amended the manuscript to better reflect the conduct of the analyses, which occurred whilst the protocol was being processed, as permitted by PROSPERO. 
Research in conxtext, evidence before this study:

"The protocol for this systematic review and meta-analysis was registered in the International Prospective Register of Systematic Reviews before the analyses were completed (PROSPERO registration number CRD42019131774).”

\section{Methods:}

"The protocol for this review was submitted before the analyses were begun (10 April 2019) and registered in the International Prospective Register of Systematic Reviews before the analyses were completed (PROSPERO registration number CRD42019131774) and can be accessed at: http://www.crd.york.ac.uk/PROSPERO/display_record.php?ID=CRD42019131774."

-No details provided as to the decision rule to proceed from systematic review to metaanalysis. While a systematic review is always possible, a meta-analysis requires an a priori decision as to what conditions (related to statistical, clinical \& methodological heterogeneity) are required.

We appreciate the reviewer's comment, and agree that the impact of methodological and statistical decisions on the conduct of systematic reviews and meta-analyses is perhaps underappreciated.

Because of the small number of eligible trials that were well known to the authors, we planned a priori to proceed from systematic review directly to meta-analysis for all dichotomous outcomes, in order to quantitatively synthesize the results and examine the consistency of treatment effects across the studies.

In contrast, we prospectively decided to summarize the long-term eGFR slope outcome descriptively. Because eGFR slope represents the absolute, rather than proportional effects of treatment, any heterogeneity in absolute effects simply reflects differences in baseline risk across the studies, and thus heterogeneity for this outcome cannot be meaningfully assessed.

After completing our main analyses, we conducted a range of post-hoc sensitivity analyses to assess the robustness (or vulnerability) of our results in response to definitional and methodological decisions.

We have clarified theses points and provided further details in the methods section:

Methods, data synthesis and analysis:

"We prespecified that results for dichotomous outcomes were to be quantitatively synthesized by individual studies using a random effects model with inverse variance weighting to obtain summary effect estimates represented as relative risk $(R R)$ with associated $95 \%$ confidence intervals $(C I)$.

"We prospectively decided to summarize the effect of SGLT2 inhibitors on long-term eGFR slope descriptively because of variations in the definition of this outcome and because it measured the absolute rather than proportional effect of treatment. Any heterogeneity between studies for this outcome therefore could not be meaningfully assessed, as differences in absolute effect reflected differences in baseline kidney risk."

"Because of the kidney-based mechanism of action and albuminuria lowering effect of SGLT2 inhibitors, we prospectively decided to conduct subgroup analyses to assess for effect modification by three kidney-related subgroups." 
"We decided a priori to use random effects meta-regression to assess trend in treatment effects across eGFR and albuminuria subgroups as the primary analysis. In sensitivity analyses we repeated the meta-regression analyses treating subgroups as categories without assumptions of linearity.”

-Patients included in the 4 trials - very tight age range (61.3 to 63.9), so findings of limited generalizability.

The mean age of participants (as quoted above) was similar across the trials; however as there was no upper age restriction for entry, these studies included a broad range of ages. One could argue that the issue of generalizability/representativeness is not limited to age (or any one particular patient characteristic) or trials of SGLT2 inhibitors, but is a general consideration of all randomized evidence, and thus we have made a more general statement to acknowledge this.

\section{Discussion:}

"The broader generalizability of these results may also be somewhat affected by the characteristics of participants in the included studies, and thus future work to assess the use of these agents in routine clinical practice will be important".

Reviewer \#3: Three CVOTs of SGLT2 inhibitors, EMPA-REG, CANVAS and DECLARE have been published over the last few years. Each of these also published their findings of a reduction in the composite kidney outcome of ESKD, worsening kidney function and death. All three showed positive kidney outome results. Not only were these data published individually for each trial but they were also then meta-analyzed in the Zelniker et al. paper in the Lancet in November last year. Unsurprisingly, given that all 3 studies showed a positive result, this was also seen in the meta-analysis and in each eGFR subgroup $(>60,60-90,<60)$.

In terms of AKI with SGLT2 inhibitors in EMPA-REG, CANVAS and DECLARE, this was also recently addressed in a meta-analysis by Gilbert et al., published earlier this year in DOM.

Most recently, we have seen the results of CREDENCE that showed a reduction in ESKD, worsening kidney function and death in patients with more advanced diabetic kidney disease.

Given the overwhelmingly positive data in each individual trial and in the Zelniker metaanalysis, it is unclear to this reviewer what additional value there is in adding the data from a dedicated DKD trial (CREDENCE) in order to undertake yet another meta-analysis.

The above concerns with regard to both ESKD/CKD progression and AKI should be precisely addressed and appropriately referenced in the introduction and in the "Evidence Before This Study" paragraph of the Research in Context section. I could not find the Zelniker or Gilbert papers referred to.

We understand that the evidence for SGLT2 inhibition in type 2 diabetes is evolving rapidly and believe the data presented in this manuscript substantially add to the literature and have direct implications for clinical practice. As discussed in our comments to the editors, we have cited these works and used them to highlight novel aspects of this meta-analysis, beyond simply the inclusion of the CREDENCE trial.

For the first time, the data clearly demonstrate that SGLT2 inhibitors reduce the risk of dialysis, transplantation or death due to kidney disease - the most important patient-centred kidney outcome. 
While this is primarily driven by the CREDENCE trial, there is no substantive evidence that the effect is not consistent across the class.

This analysis, now with complete data for all outcomes following the publication of the DECLARE-TIMI 58 renal outcomes last week, is especially timely since the use of SGLT2 inhibitors in patients with eGFR $<45$ is not currently permitted in most countries. Perhaps most importantly, the clear evidence of benefit for patients with starting eGFR $<45 \mathrm{~mL} / \mathrm{min} / 1.73 \mathrm{~m}^{2}$ demonstrated for the first time in this analysis suggests that many more individuals are likely to benefit from treatment if current restrictions are reconsidered.

Additionally, whether baseline albuminuria modifies the effect of SGLT2 inhibition on kidney outcomes has not been clear, and until recently too few participants with higher levels of albuminuria have been studied to robustly assess effect modification.

As suggested, we have explicitly outlined in the research in context, introduction and discussion sections what questions this meta-analysis addresses that have not been answered in previous works.

Research in context, evidence before this study:

"SGLT2 inhibitors are not currently approved for use in patients with estimated glomerular filtration rate (eGFR) less than 45 or $60 \mathrm{~mL} / \mathrm{min} / 1.73 \mathrm{~m}^{2}$ in most countries, primarily because their glucose lowering effect is substantially dependent on kidney function. A recent meta-analysis of these trials has reported that the renoprotective effect of SGLT2 attenuates with declining kidney function. However, less than a sixth of participants studied had a baseline eGFR less than 60 $\mathrm{mL} / \mathrm{min} / 1 \cdot 73 \mathrm{~m}^{2}$, and even fewer had baseline eGFR less than $45 \mathrm{~mL} / \mathrm{min} / 1 \cdot 73 \mathrm{~m}^{2}$. The ability to robustly assess effects in people with reduced kidney function was therefore limited, especially since few patient-level kidney outcomes occurred. While collectively these trials have suggested that SGLT2 inhibitors might protect against acute kidney injury, the safety of these agents in patients at high risk of adverse kidney outcomes has remained a concern."

Introduction:

"A recent meta-analysis of cardiovascular outcome trials suggested that the effect of SGLT2 inhibitors on kidney outcomes attenuates with declining eGFR. However, less than a sixth of participants in this analysis had baseline eGFR $<60 \mathrm{~mL} / \mathrm{min} / 1.73 \mathrm{~m} 2$, and thus the ability to robustly assess effect modification by kidney function has been limited. Additionally, because very few participants with starting eGFR $<45 \mathrm{~mL} / \mathrm{min} / 1.73 \mathrm{~m} 2$ have been studied, it has also been unclear whether these patients derive protection against kidney outcomes, since the glycaemic efficacy of these agents is substantially attenuated in this population. Similarly, few participants with higher levels of albuminuria have been studied, and therefore the consistency of treatment effect across different levels of albuminuria is unclear. While these trials have collectively suggested protection against acute kidney injury, the safety of these agents in patients at higher risk of adverse kidney outcomes has not been well established."

\section{Discussion:}

"A recent meta-analysis of the cardiovascular outcome trials suggested that the renoprotective effect of SGLT2 inhibitors attenuates with declining kidney function. However too few participants with baseline eGFR $<60 \mathrm{~mL} / \mathrm{min} / 1.73 \mathrm{~m}^{2}$ were studied to adequately assess trend by eGFR. Additionally, whether participants with starting eGFR $<45 \mathrm{~mL} / \mathrm{min} / 1 \cdot 73 \mathrm{~m}^{2}$ derive benefits with respect to kidney outcomes, despite substantially attenuated glycaemic efficacy, has also been unclear."

Other concerns relate to missing data. 
1. DECLARE data are not included due to what is described as lack of granularity. Given that DECLARE data are missing from Figure 1, the study's primary analysis, this issue should be resolved.

What efforts were undertaken to remedy this lack of data? Given that DECLARE was only published in November 2018, will these data appear in the TIMI group's own sub-analysis? If this is the case, then the present meta-analysis might be viewed as too early.

We have updated all the analyses following the recent publication of the DECLARE-TIMI 58 renal outcomes manuscript. There is now complete outcome data for all outcomes, including the primary outcome, as well as subgroup analyses by baseline eGFR, albuminuria and use of renin-angiotensin system blockade.

We have updated our analyses accordingly.

2. Event rates from participants in EMPA-REG Outcome are missing throughout. These data should be obtained if at all possible. Have the authors approached the EMPA-REG investigators for this? Is it being withheld? Is it available through the FDA?

As outlined in our response to the editors, we received data on the primary outcome from the DECLARE-TIMI 58 investigators during the revision of the manuscript. These data have not been previously published, including in the recent DECLARE-TIMI 58 renal outcomes paper which was published in this Journal last week.

We approached the EMPA-REG OUTCOME investigators and trial sponsors as part of this systematic review and meta-analysis. The cardio-renal composite outcome of substantial loss of kidney function, ESKD, or death due to cardiovascular or kidney disease in the EMPA-REG OUTCOME trial has not been previously published and was obtained through correspondence with the trial sponsor (Figure 2, panel C). Additionally the number of events for the primary outcome of dialysis, transplantation or death due to kidney disease in the EMPA-REG OUTCOME trial was also clarified with the trial sponsor.

Unfortunately event rates per 1000 patient years are variably reported across the primary trial reports and secondary analyses and we were not able to obtain event rates for all studies. We are currently unaware of any reporting of event rates from the EMPA-REG OUTCOME trial for eGFR and UACR subgroups despite an extensive search.

In the CANVAS Program publications, event rates have been reported but the number of events in each trial arm has not been for statistical reasons. This is because the CANVAS Program comprised two parallel companion trials that were analyzed and reported as one integrated dataset. Because of the combined effect of the differences in randomization ratios and differences in follow-up duration between the trials, the number of events/number of participants in canagliflozin and placebo arms cannot be used to derive risk ratios, as these will give erroneous effect estimates, and thus have never been reported.

To address this, we have reported the total number of events and participants in each trial for every outcome so that the figures are as complete as possible.

3. DECLARE data are also missing from the analysis according to UACR. Once again, what efforts been made to obtain these data or are they pending disclosure at ADA or ASN later this year, in which case the submitted meta-analysis is just too early. 
With the recent publication of the DECLARE-TIMI 58 renal outcomes paper in this Journal, we have updated the subgroup analysis by baseline UACR. This analysis now includes complete data from all four studies. We have updated Figure 3 accordingly.

Results, subgroup analysis:

"The effect on the outcome of substantial loss of kidney function, ESKD or death due to kidney disease was reported according to eGFR and UACR subgroups, and according to baseline use of RAS blockade in all four studies."

Reviewer \#4: The analysis was well done by this group of experienced trialists and epidemiologists. I have 2 comments:

*The definitions of kidney failure (as the need for chronic dialysis or kidney transplantation, or death due to kidney disease) and ESKD (as chronic dialysis, kidney transplantation, or sustained eGFR less than $15 \mathrm{~mL} / \mathrm{min} / \mathbf{1} \cdot 73 \mathrm{~m} 2$ ) are confusing. The former can be recategorized as RRT/renal death.

As suggested, we have renamed our primary outcome as "dialysis, transplantation or death due to kidney disease" to distinguish it from end-stage kidney disease. Our full description of changes to the manuscript is outlined above in detailed comments a similar query from another reviewer.

*Although it was pointed out that "a single trial contributed a substantial proportion of the data using a single agent, conducted in the population at highest risk of kidney failure", it would be better to explicitly state this as a limitation in that a substantial proportion of the renal outcome data was derived from a single study (CREDENCE) using canagliflozin, thus limiting the generalizability of this analysis to other SGLT2is.

We have amended the manuscript as suggested.

Discussion:

"A substantial proportion of the data were derived from a single study of canagliflozin (CREDENCE trial) that was stopped early, which may increase the risk of overestimating treatment effects, however the consistency with the results from the other trials reduces that risk, and is an important finding of this review. The consistency of effects among other members of the class therefore remains somewhat uncertain although there is currently no evidence of substantive heterogeneity."

Reviewer \#5: This paper reports results of a systematic review and meta-analysis of cardiovascular outcomes trials and a kidney outcome trial for effect of SGLT2 inhibitors on kidney failure in patients with type 2 diabetes. Kidney failure was defined as chronic dialysis, transplant, or death due to kidney disease. Data from a total of 38,723 clinical trial participants were used for these analyses. Overall, risk of kidney failure was reduced by almost $30 \%$ and AKI was decreased by $25 \%$. The benefit of SGLT2 inhibitors on kidney failure may attenuate with lower eGFR. Notably, effects of SGLT2 inhibitors on substantial loss of kidney function, end-stage kidney disease, or death due to kidney disease was observed irrespective of renin angiotensin system blockade.

Knowledge about effects of SGLT2 inhibitors on risk of kidney failure in patients with type 2 diabetes is essential to optimal care. Since kidney failure clearly influences how people feel, function, and survive, such data are central to deploying therapeutic approaches for patientdriven priorities. Overall, this is an expertly conducted and impactful study. 
I offer the following comments for the authors to consider in their revision:

Major

1. The possibility that benefit of SGLT2 inhibitors may attenuate with lower eGFR has been appropriately framed and called-out. However, it may be worthwhile to acknowledge that other classes of agents, e.g. GLP-receptor agonists, effectively and safely treat hyperglycemia and slow eGFR decline with baseline eGFR as low as $15 \mathrm{~mL} / \mathrm{min} / 1.73 \mathrm{~m} 2$. As such, future studies might evaluate drug combinations or compare SGLT2 inhibitors with other agents based upon particular clinical profiles like advanced CKD.

We appreciate the reviewers comment and have made note of this in the discussion section.

Discussion:

"Future studies evaluating the combination of SGLT2 inhibitors with other glucose lowering agents that have shown beneficial impacts on kidney function (such as glucagon-like receptor agonists) are another potential area of interest, particularly in patients with established diabetic kidney disease."

2. A striking, admittedly exploratory finding, was that renin angiotensin system blockade may not have much effect on the composite of substantial loss of kidney function, end-stage kidney disease, or death due to kidney disease when SGLT inhibitors are also used. Might such data provide rationale for study of SGLT inhibitors without background renin angiotensin system blockade for treatment in some groups of patients, e.g. non-albuminuric DKD or others? Consider moving this data to the main paper in Figure 3 where potential effect modifiers are presented.

With the addition of data from the DECLARE-TIMI 58 trial, we have complete data on the composite renal outcome by baseline RAS blockade across all four studies. As suggested, we have moved this data to the main paper in Figure 3. The lack of effect modification by UACR and consistent benefits in patients irrespective of the use of renin-angiotensin system blockade indicate that SGLT2 inhibitors should provide benefit for a broader patient population. We have edited the manuscript to highlight this point.

Discussion:

"The absence of effect modification by baseline albuminuria contrasts with the findings from trials of RAS blockade. These data suggest that mechanisms other than those associated with albuminuria reduction might also be important. Furthermore, renoprotection with SGLT2 inhibitors was consistent irrespective of baseline use of RAS blockade. Taken together, these findings suggest that SGLT2 inhibition should provide benefit for a broader patient population."

\section{Minor}

1. In the Abstract, the distinction between "kidney failure" and "ESKD" would benefit from clearer explanation.

As outlined in our response to the editors and other reviewers, we have clarified terminology of the different outcomes so that our primary outcome is "dialysis, transplantation or death due to kidney disease" which is more clearly distinct from "end-stage kidney disease". Our full response to this suggestion is detailed above. 


\section{Table 1. Characteristics of included studies}

eGFR: estimate glomerular filtration rate; MDRD: modification of diet in renal disease equation;

$\mathrm{CrCl}$ : creatinine clearance; CKD-EPI: chronic kidney disease epidemiology collaboration equation;

UACR: urinary albumin:creatinine ratio; RAS: renin-angiotensin system blockade; N/A: not available

\begin{tabular}{|c|c|c|c|c|}
\hline Study & $\begin{array}{l}\text { EMPA-REG } \\
\text { OUTCOME } \\
(n=7020)\end{array}$ & $\begin{array}{l}\text { CANVAS } \\
\text { Program } \\
(n=10142)\end{array}$ & $\begin{array}{l}\text { DECLARE- } \\
\text { TIMI } 58 \\
(\mathrm{n}=17160) \\
\end{array}$ & $\begin{array}{l}\text { CREDENCE } \\
(n=4401)\end{array}$ \\
\hline Drug & Empagliflozin & Canagliflozin & Dapagliflozin & Canagliflozin \\
\hline Dose & $\begin{array}{l}10 \mathrm{mg} \text { and } \\
25 \mathrm{mg}\end{array}$ & $\begin{array}{l}100 \mathrm{mg} \text { and } \\
300 \mathrm{mg}\end{array}$ & $10 \mathrm{mg}$ & $100 \mathrm{mg}$ \\
\hline Number of participants & 7020 & 10142 & 17160 & 4401 \\
\hline Mean age (years) & $61 \cdot 3$ & $63 \cdot 3$ & $63 \cdot 9$ & $63 \cdot 0$ \\
\hline Female, n (\%) & $2004(28 \cdot 5)$ & $3633(35 \cdot 8)$ & $6422(37 \cdot 4)$ & $1494(33 \cdot 9)$ \\
\hline Median follow-up (years) & $3 \cdot 1$ & $2 \cdot 4$ & $4 \cdot 2$ & $2 \cdot 6^{\S}$ \\
\hline eGFR inclusion criteria & $\geq 30$ (MDRD) & $\geq 30(\mathrm{MDRD})$ & $\begin{array}{l}\mathrm{CrCl} \geq 60 \\
\mathrm{~mL} / \mathrm{min} \\
\text { (Cockcroft- } \\
\text { Gault) }\end{array}$ & $\begin{array}{l}30-<90(\mathrm{CKD}- \\
\mathrm{EPI})\end{array}$ \\
\hline \multicolumn{5}{|l|}{$\begin{array}{l}\mathrm{eGFR}, \mathrm{mL} / \mathrm{min} / 1 \cdot 73 \mathrm{~m}^{2}, \mathrm{n} \\
(\%)^{* \dagger}\end{array}$} \\
\hline$\geq 90$ & $1538(21 \cdot 9)$ & $2476(24 \cdot 4)$ & $8162(47 \cdot 6)$ & - \\
\hline $60-<90$ & $3661(52 \cdot 2)$ & $5625(55 \cdot 5)$ & $7732(45 \cdot 1)$ & $1809(41 \cdot 1)$ \\
\hline $45-<60$ & $1249(17 \cdot 8)$ & $1485(14 \cdot 6)$ & $1265^{\mathbb{I}}(7 \cdot 4)$ & $1279(29 \cdot 1)$ \\
\hline$<45$ & $570(8 \cdot 1)$ & $554(5 \cdot 5)$ & N/A & $1313(29 \cdot 8)$ \\
\hline UACR criteria, mg/g & None & None & None & $>300$ to 5000 \\
\hline \multicolumn{5}{|l|}{ UACR mg/g, n $(\%)^{\dagger}$} \\
\hline$<30$ & $4142(59 \cdot 0)$ & $7007(69 \cdot 1)$ & $11644(67 \cdot 9)$ & - \\
\hline $30-300$ & $1996(28 \cdot 4)$ & $2266(22 \cdot 3)$ & $4030(23 \cdot 5)$ & - \\
\hline$>300$ & $764(10 \cdot 9)$ & $760(7 \cdot 5)$ & $1169(6 \cdot 8)$ & $4401(100 \cdot 0)$ \\
\hline $\begin{array}{l}\text { Baseline use of RAS } \\
\text { blockade, } \mathrm{n}(\%)\end{array}$ & $5666(80 \cdot 7)$ & $8116(80 \cdot 0)$ & $13950(81 \cdot 3)$ & 4395 (99.9) \\
\hline
\end{tabular}


*Based on the MDRD equation in EMPA-REG OUTCOME and the CANVAS Program and the CKD-EPI equation in DECLARE-TIMI 58 and CREDENCE.

IIIncludes all DECLARE-TIMI58 participants with eGFR $<60 \mathrm{~mL} / \mathrm{min} / 1 \cdot 73 \mathrm{~m}^{2}$

$\S$ Stopped early after a planned interim analysis on the recommendation of the Independent Data Monitoring Committee

$\uparrow$ Based on screening eGFR and UACR measurements in the CREDENCE trial 
Figure 1. Effect of SGLT2 inhibitors on dialysis, transplantation or death due to kidney disease

SGLT2: sodium-glucose cotransporter 2 inhibitor; RR: relative risk; CI: confidence interval.

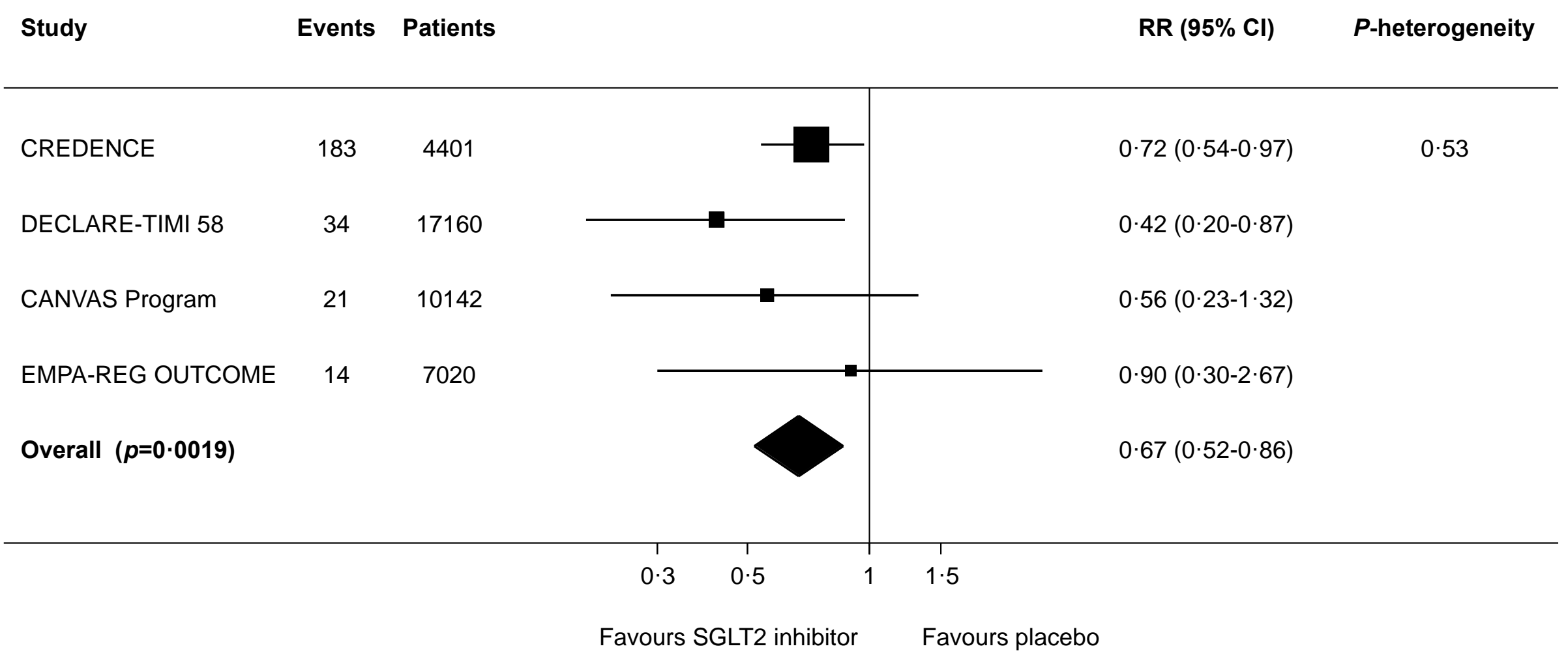

Weights were from random effects meta-analysis; corresponding $\mathrm{I}^{2}=0.0 \%$ 
Figure 2. Effects of SGLT2 inhibitors on (A) ESKD", (B) substantial loss of kidney function ${ }^{\S}$, ESKD, or death due to kidney disease and (C) substantial loss on kidney function, ESKD, or death due to cardiovascular or kidney disease

ESKD: end-stage kidney disease; SGLT2: sodium-glucose cotransporter 2 inhibitor; RR: relative risk; CI: confidence interval.

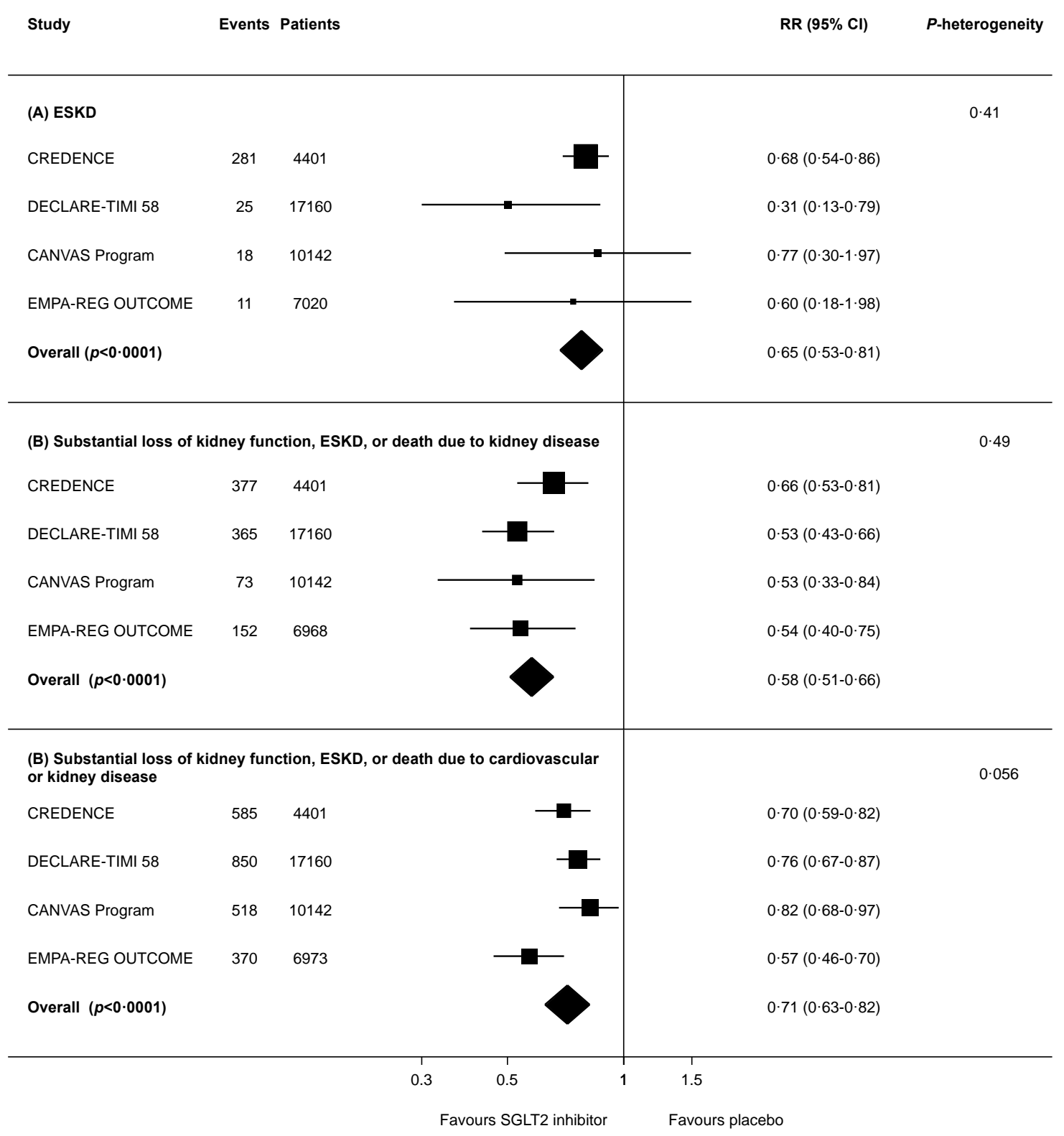

Weights were from random effects meta-analysis. $\mathrm{I}^{2}=0.0 \%$ for $\mathrm{ESKD}, \mathrm{I}^{2}=0.0 \%$ for substantial loss of kidney function, ESKD or death due to kidney disease, and $\mathrm{I}^{2}=60.3 \%$ for substantial loss of kidney function, ESKD or death due to cardiovascular or kidney disease. 
II ESKD was defined as chronic dialysis, transplantation, or sustained eGFR $<15 \mathrm{~mL} / \mathrm{min} / 1.73 \mathrm{~m}^{2}$, except in the EMPA-REG OUTCOME trial where it was defined as chronic dialysis or transplantation.

$\S$ Substantial loss of kidney function was defined as doubling of serum creatinine, except in the DECLARE-TIMI 58 trial, where it was defined as sustained $40 \%$ decline in eGFR 
Figure 3. Effect of SGLT2 inhibitors on substantial loss of kidney function ${ }^{\mathbb{T}}$, ESKD, or death due to kidney disease, stratified by baseline (A) eGFR ${ }^{\S}$, (B) UACR ${ }^{\mathrm{II}}$, and (C) use of RAS blockade ${ }^{\dagger}$

ESKD: end-stage kidney disease; eGFR: estimated glomerular filtration rate; UACR: urinary albumin:creatinine ratio; RAS: renin-angiotensin system; SGLT2: sodium-glucose cotransporter 2 inhibitor; RR: relative risk; CI: confidence interval.

II Substantial loss of kidney function was defined as doubling of serum creatinine, except in the DECLARE-TIMI 58 trial, where it was defined as sustained $40 \%$ decline in eGFR

$\S$ Results from the CREDENCE trial based on screening eGFR and UACR measurements

$\dagger$ Use of RAS blockade was mandated as part of entry into the CREDENCE trial 
(A) eGFR $\geq 90 \mathrm{~mL} / \mathrm{min} / 1.73 \mathrm{~m}^{2}: \mathrm{I}^{2}=41.8 \%, P$-heterogeneity=0.18; eGFR $60-<90 \mathrm{~mL} / \mathrm{min} / 1.73 \mathrm{~m}^{2}: \mathrm{I}^{2}=0.0 \%, P$-heterogeneity=0.46; eGFR 45 - $<60$ $\mathrm{mL} / \mathrm{min} / 1.73 \mathrm{~m}^{2}: \mathrm{I}^{2}=0.0 \%, P$-heterogeneity $=0.52 ;$ eGFR $<45 \mathrm{~mL} / \mathrm{min} / 1.73 \mathrm{~m}^{2}: \mathrm{I}^{2}=0.0 \%, P$-heterogeneity $=0.94$

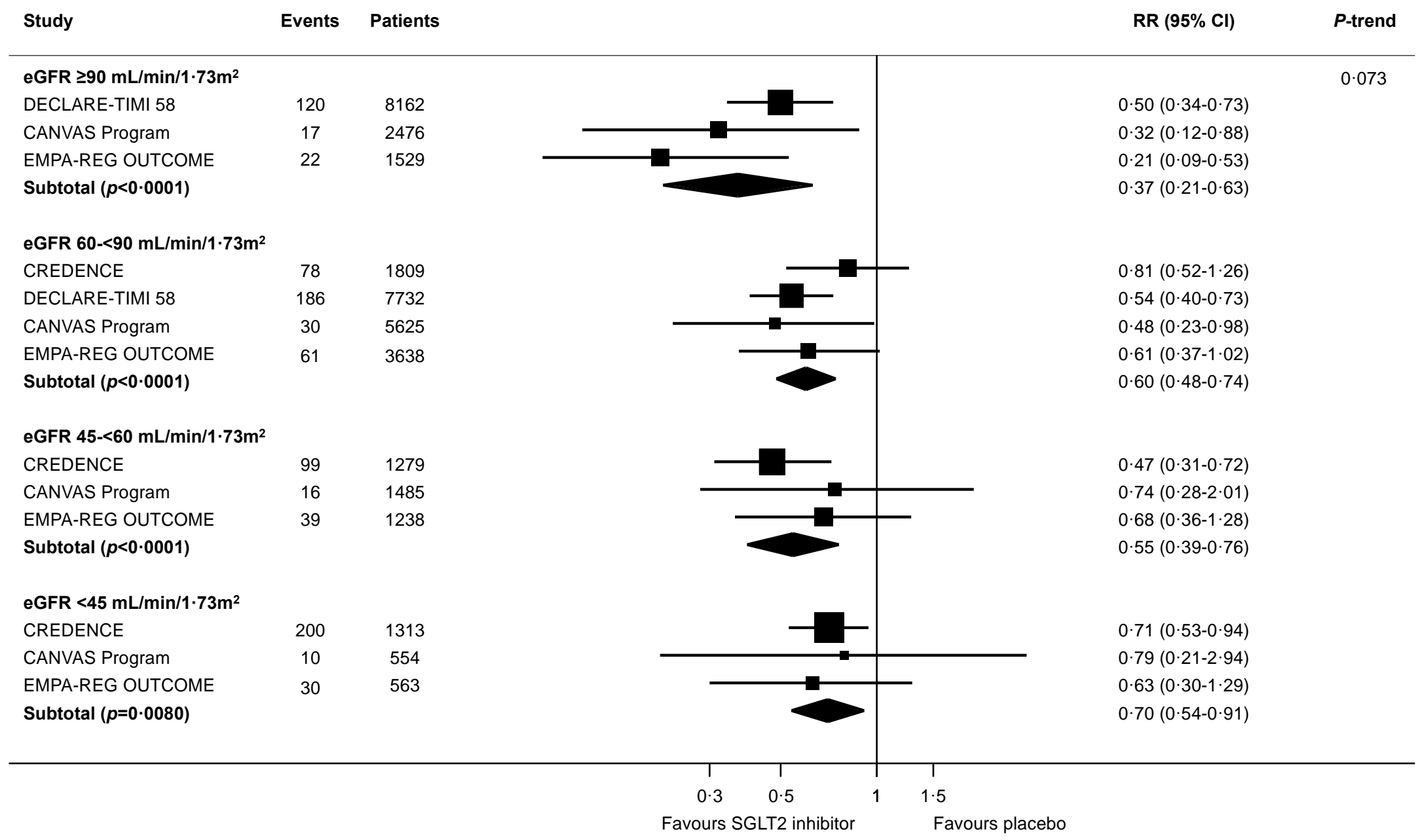


(B) UACR <30 mg/g: $\mathrm{I}^{2}=10.3 \%, P$-heterogeneity=0.33; UACR 30-300 mg/g: $\mathrm{I}^{2}=18.5 \%, P$-heterogeneity=0.29, UACR >300 mg/g: $\mathrm{I}^{2}=51.0 \%, P$ -

heterogeneity $=0.11$

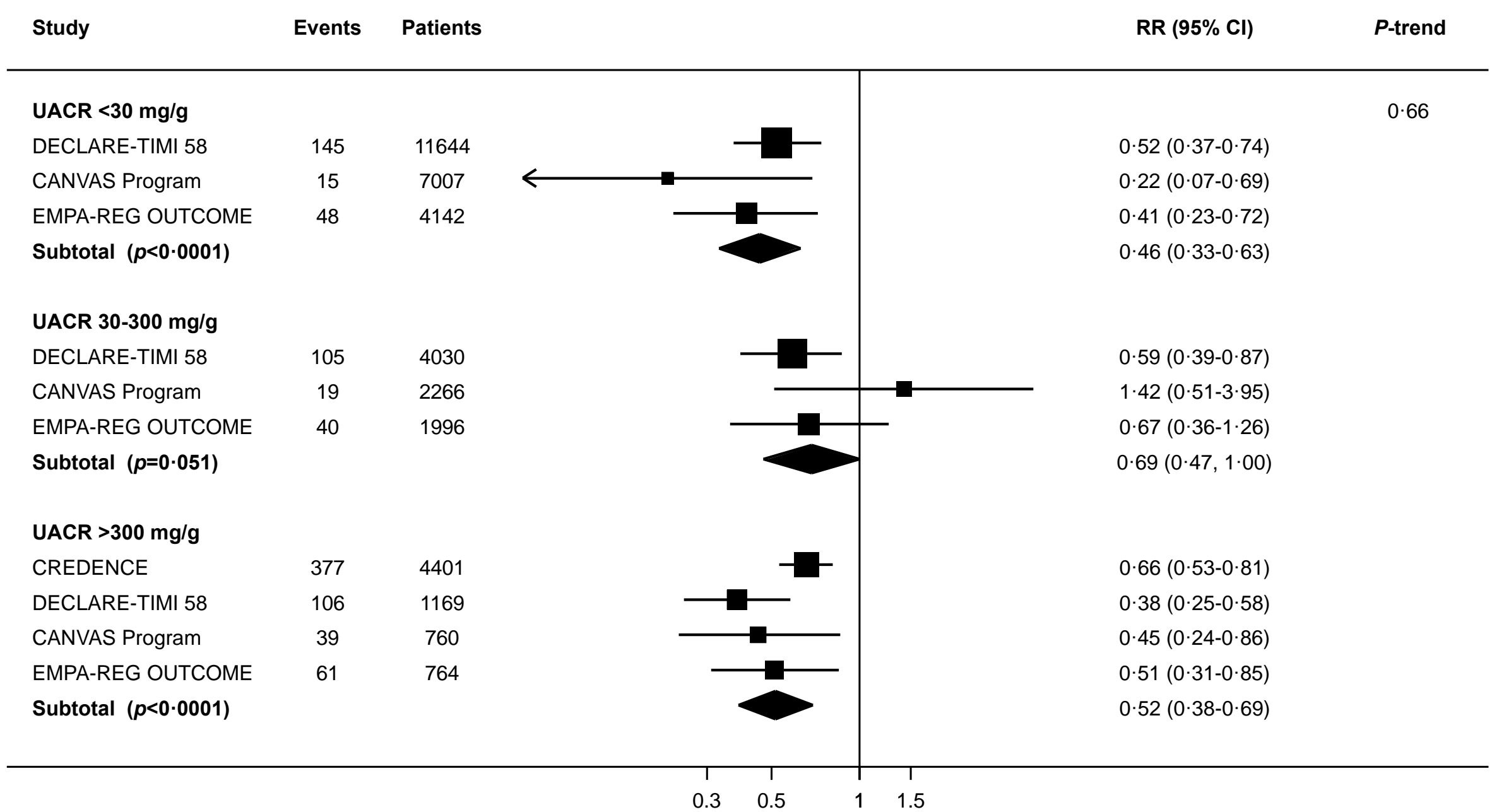


(C) RAS blockade: $\mathrm{I}^{2}=8.7 \%, P$-heterogeneity $=0.35$; No RAS blockade: $\mathrm{I}^{2}=0.0 \%, P$-heterogeneity $=0.92$

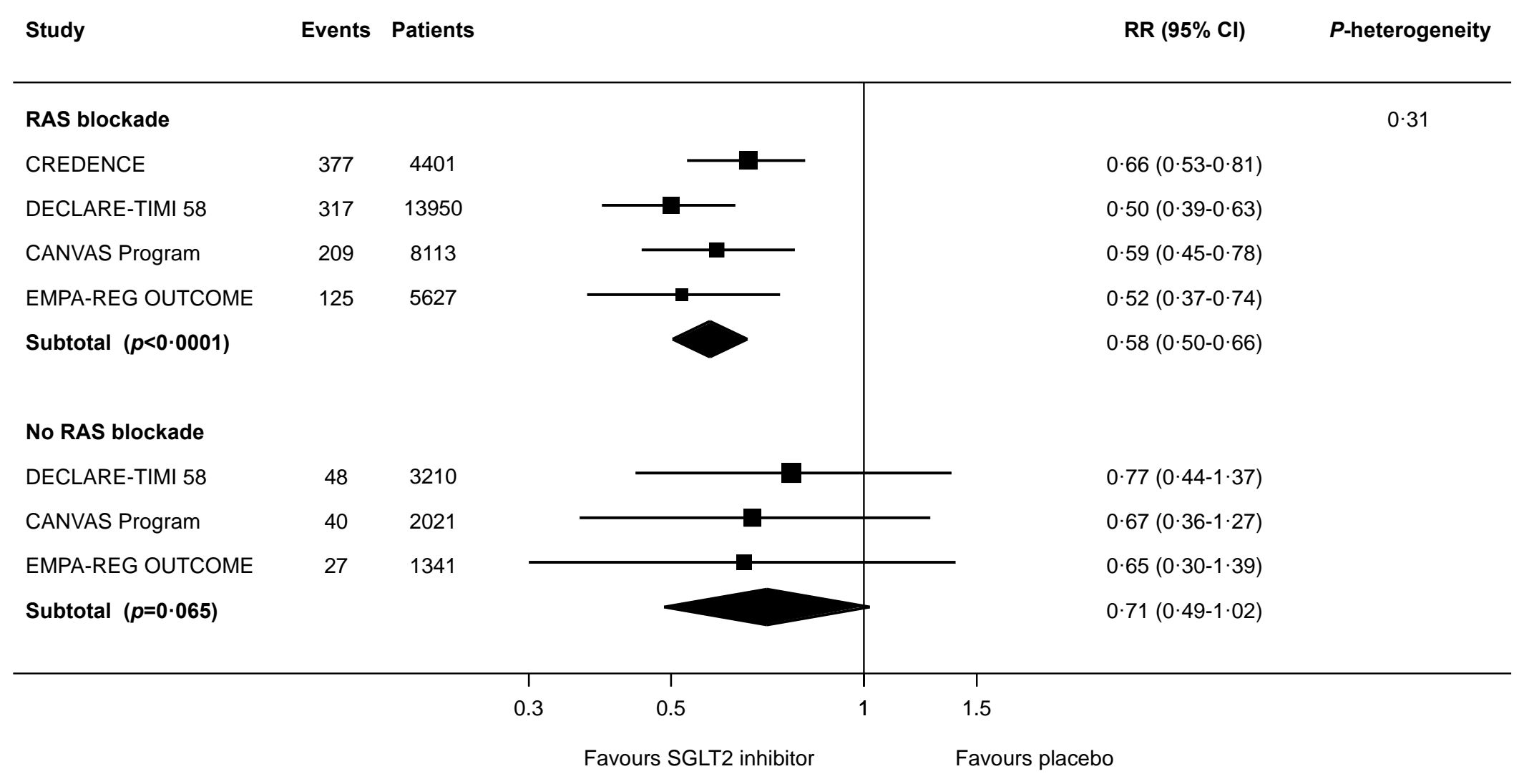

Weights were from random effects meta-analysis. $P$-trend values across eGFR and UACR subgroups were calculated against a t-distribution in random effects meta-regression using restricted maximum likelihood with Hartung Knapp modification. $P$-heterogeneity for between subgroup differences in (C) was obtained from a random effects model. 
Figure 4. Effect of SGLT2 inhibitors on acute kidney injury

SGLT2: sodium-glucose cotransporter 2 inhibitor; RR: relative risk; CI: confidence interval.

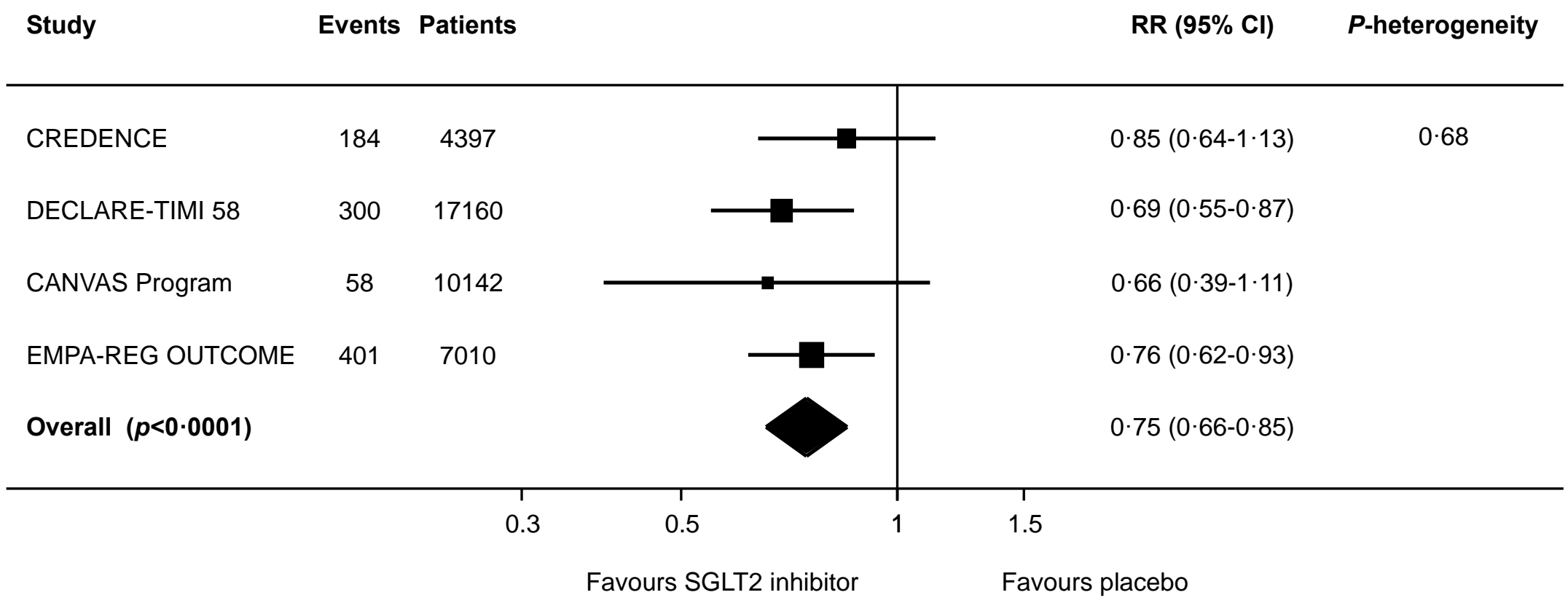

Weights were from random effects meta-analysis; corresponding $\mathrm{I}^{2}=0.0 \%$ 


\section{Figure 5. Summary of the effects of SGLT2 inhibition on major kidney outcomes}

ESKD: end-stage kidney disease; AKI: acute kidney injury; SGLT2: sodium-glucose cotransporter 2 inhibitor; RR: relative risk; CI: confidence interval.

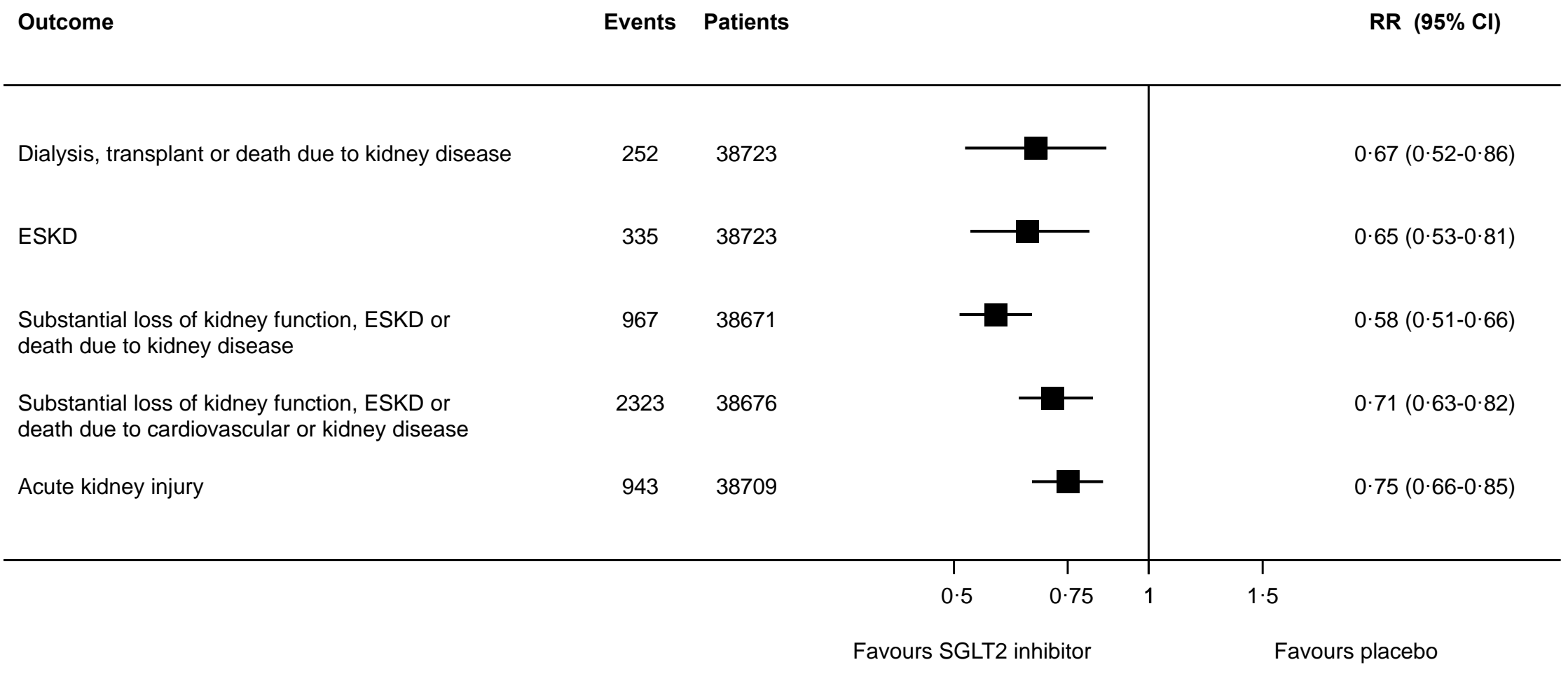


Supplementary appendix
Click here to download Necessary Additional Data: Supplementary appendix_LDE_1.pdf

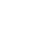

$\sqrt{10}$

$\sqrt{2}$

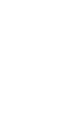
更 更 Click here to dow uread Necessary Additional Data: Supplementary appendix_LDE_.pd (1)

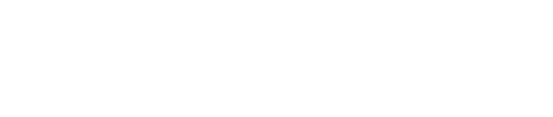
. . . . 列 . . . 列 . 列 列 列 列

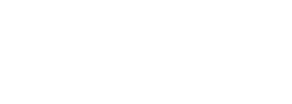


PRISMA checklist
Click here to download Necessary Additional Data: PRISMA_checklist_LDE.doc

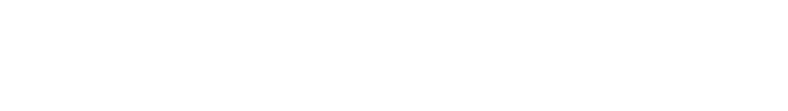

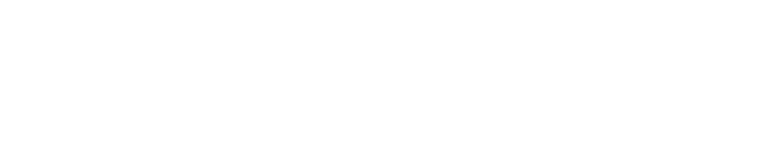

